\title{
Mapping Extracellular pH of Gliomas in Presence of Superparamagnetic Nanoparticles: Towards Imaging the Distribution of Drug-Containing Nanoparticles and Their Curative Effect on the Tumor Microenvironment
}

\author{
Samuel Maritim, ${ }^{1,2}$ Daniel Coman, ${ }^{1,3}$ Yuegao Huang, ${ }^{1,3}$ Jyotsna U. Rao, ${ }^{1,3}$ \\ John J. Walsh, ${ }^{1,2}$ and Fahmeed Hyder ${ }^{1,2,3}$ \\ ${ }^{1}$ Magnetic Resonance Research Center, Yale University, New Haven, CT, USA \\ ${ }^{2}$ Department of Biomedical Engineering, Yale University, New Haven, CT, USA \\ ${ }^{3}$ Department of Radiology and Biomedical Imaging, Yale University, New Haven, CT, USA
}

Correspondence should be addressed to Samuel Maritim; samuel.maritim@yale.edu and Fahmeed Hyder; fahmeed.hyder@yale.edu Received 11 July 2017; Revised 25 September 2017; Accepted 3 October 2017; Published 22 November 2017

Academic Editor: Maria P. Morales

Copyright (c) 2017 Samuel Maritim et al. This is an open access article distributed under the Creative Commons Attribution License, which permits unrestricted use, distribution, and reproduction in any medium, provided the original work is properly cited.

\begin{abstract}
Since brain's microvasculature is compromised in gliomas, intravenous injection of tumor-targeting nanoparticles containing drugs (D-NPs) and superparamagnetic iron oxide (SPIO-NPs) can deliver high payloads of drugs while allowing MRI to track drug distribution. However, therapeutic effect of D-NPs remains poorly investigated because superparamagnetic fields generated by SPIO-NPs perturb conventional MRI readouts. Because extracellular $\mathrm{pH}\left(\mathrm{pH}_{\mathrm{e}}\right)$ is a tumor hallmark, mapping $\mathrm{pH}_{\mathrm{e}}$ is critical. Brain $\mathrm{pH}_{\mathrm{e}}$ is measured by biosensor imaging of redundant deviation in shifts (BIRDS) with lanthanide agents, by detecting paramagnetically shifted resonances of nonexchangeable protons on the agent. To test the hypothesis that $\mathrm{BIRDS}^{-b a s e d ~} \mathrm{pH}_{\mathrm{e}}$ readout remains uncompromised by presence of SPIO-NPs, we mapped $\mathrm{pH}_{\mathrm{e}}$ in glioma-bearing rats before and after SPIO-NPs infusion. While SPIO-NPs accumulation in the tumor enhanced MRI contrast, the $\mathrm{pH}_{\mathrm{e}}$ inside and outside the MRI-defined tumor boundary remained unchanged after SPIO-NPs infusion, regardless of the tumor type (9L versus RG2) or agent injection method (renal ligation versus coinfusion with probenecid). These results demonstrate that we can simultaneously and noninvasively image the specific location and the healing efficacy of D-NPs, where MRI contrast from SPIO-NPs can track their distribution and BIRDSbased $\mathrm{pH}_{\mathrm{e}}$ can map their therapeutic impact.
\end{abstract}

\section{Introduction}

Treatment and management of glioblastoma, the most common and malignant form of primary brain tumors, represent an unmet clinical challenge [1]. While gliomas are relatively rare compared to other forms of cancer malignancies [1], they are characterized by the worst prognosis, with a 5year survival of less than 10\% [2]. Treatments fail because gliomas are highly invasive, the blood brain barrier (BBB) prevents drugs from reaching the tumor at therapeutic doses, and systemic toxicity limits benefits from therapy [3-5]. In addition, there is a lack of reliable in vivo methods that can simultaneously and noninvasively measure the delivery and therapeutic benefits of cancer drugs. Therapy can be greatly improved by delivering high drug doses specifically to the tumor (while minimizing systemic toxicities) and by timely and quantitative monitoring of the delivery and efficacy of these drugs.

The transport and delivery of therapeutic agents into the brain parenchyma are impeded by a dense network of capillary endothelial cells, pericytes, and perivascular macrophages, which together form the BBB [6]. In the healthy brain, the $\mathrm{BBB}$ allows a highly selective transport of endogenous substances (e.g., nutrients) that are critical to brain function 
while keeping out potentially harmful toxins and drugs that are circulating in the blood $[7,8]$. However, the BBB is disrupted in several pathologies including high-grade gliomas leading to increased leakiness (i.e., hyperpermeability) $[9,10]$. Breakthroughs in glioma imaging and chemotherapy exploit the fact that nanoparticles (NPs) loaded with drugs (DNPs) and MRI contrast agents like superparamagnetic iron oxide (SPIO-NPs) can extravasate from the blood through the large vascular fenestrations into the tumor [11, 12]. The combination of increased vascular permeability and poor lymphatic clearance in tumors leads to accumulation of NPs in tumors through enhanced permeation and retention (EPR) [13]. While tumors, including gliomas, generally possess larger vascular fenestrations (and hence higher permeability) compared to healthy tissue, these fenestrations are highly dependent on the location of the vessels in the tumor (i.e., fewer abnormalities in vessels on tumor periphery and higher in the tumor core), and moreover the fenestrations depend on the age/size of the tumor (i.e., larger tumors tend to have more abnormalities) [14, 15]. The pores on tumor vasculature include caveolae, vesiculo-vacuolar organelles, and fenestrations that are on the order of $10-20 \mathrm{~nm}$ along with larger but sporadic interendothelial cell gaps, which are significantly larger than $200 \mathrm{~nm}$ in diameter [16-20]. Thus NPs like the Molday ION (or SPIO-NPs; $30-50 \mathrm{~nm}$ hydrodynamic diameter) can extravasate passively across the $\mathrm{BBB}$ of the tumor niche more effectively compared to the normal neuropil. Therefore extravasation and accumulation of NPs will vary between the tumor core, tumor boundary, and healthy tissue [21-25].

Tumor-specific delivery of D-NPs can be further enhanced by coating the D-NPs with ligands that target overexpressed receptors and/or transporters in tumors [26-29]. Despite these advances in targeting of D-NPs for delivering high drug payloads to tumors, the effect of these $\mathrm{D}-\mathrm{NPs}$ on the tumor microenvironment remains largely unknown. SPIO-NPs have been evaluated and approved for clinical use as MRI contrast agents [30-37]. Because the MRI contrast generated by SPIO-NPs persists for a long time, SPIO-NPs have recently been combined with D-NPs and used to simultaneously image drug delivery and biodistribution with MRI [12, 38-41]. However, the large superparamagnetic fields generated by SPIO-NPs disturb most MRI molecular readouts.

Because low extracellular $\mathrm{pH}\left(\mathrm{pH}_{\mathrm{e}}\right)$ is a hallmark of cancer pathogenesis and promotes tumor invasion and resistance to therapy [42-48], there is need for advanced $\mathrm{pH}_{\mathrm{e}}$ mapping methods to enable monitoring of glioma invasion. Since some drugs only work in certain $\mathrm{pH}$ ranges, precise knowledge of $\mathrm{pH}_{\mathrm{e}}$ can aid in choosing and tailoring therapeutic regimens [49-51]. Additionally, their therapeutic efficacy may be assessed by measuring their ability to raise and normalize $\mathrm{pH}_{\mathrm{e}}$, for example, by drugs that alter $\mathrm{pH}_{\mathrm{e}}$ directly or affect tumor's aerobic glycolysis. Many MRI methods exist for measuring and mapping $\mathrm{pH}_{\mathrm{e}}$. Relaxation-based methods (e.g., with $\mathrm{Gd}^{3+}$ ) are highly dependent on the degree of tissue perfusion and local agent concentration thus making quantification of $\mathrm{pH}_{\mathrm{e}}$ difficult [52]. $\mathrm{pH}_{\mathrm{e}}$-sensitive MRI methods based on proton exchange (i.e., between water protons and protons of amide/amine and hydroxyl moieties) such as chemical exchange saturation transfer (CEST) are also dependent on agent concentration and may additionally be complicated by magnetization transfer effects [53]. Spectroscopic methods, for example, ${ }^{31} \mathrm{P}$ MRS with 3-aminopropyl phosphonate (3APP), which have $\mathrm{pH}_{\mathrm{e}}$-sensitive exchangeable protons [54, 55], suffer from low spatial resolution and significant line broadening in the presence of SPIO-NPs [56].

We previously obtained $\mathrm{pH}_{\mathrm{e}}$ maps in glioma-bearing rats with biosensor imaging of redundant deviation in shifts (BIRDS) using lanthanide agents, for example, thulium 1,4,7,10-tetraazacyclododecane-1,4,7,10-tetrakis (methylene phosphonate), TmDOTP ${ }^{5-}[57,58]$. Since the BIRDS platform is based on direct detection of the paramagnetically shifted resonances of the nonexchangeable protons on the agents (rather than their peak amplitude or effect on water relaxation rate), the $\mathrm{pH}_{\mathrm{e}}$ readout with BIRDS is independent of agent concentration $[59,60]$. The functional part of the $\mathrm{pH}$ sensitivity stems from the $\mathrm{pH}$-sensitive exchangeable protons of the phosphonate groups on the agents. With advanced $\mathrm{k}$-space sampling of ultra-fast chemical shift imaging (CSI), the spatiotemporal resolution of BIRDS has improved [61]. Previously we observed in vitro that the $\mathrm{pH}$ sensitivities and readout with BIRDS agents are not compromised by the presence of SPIO-NPs [62]. Here we hypothesized that BIRDS-based $\mathrm{pH}_{\mathrm{e}}$ readout in glioma-bearing rats remains uncompromised by the presence of SPIO-NPs. We compared $\mathrm{pH}_{\mathrm{e}}$ measured with $\operatorname{TmDOTP}^{5-}$ by BIRDS before and after infusion of SPIO-NPs in rats bearing 9L gliosarcomas and RG2 gliomas. We used different agent administration methods (renal ligation versus coinfusion with probenecid) to inhibit the rapid clearance of the agent by the renal system. In addition, we compared the transverse relaxation rate enhancement from SPIO-NPs across brain regions. Our results suggest that we can use the MRI contrast from SPIO-NPs to track the distribution of D-NPs and then use the BIRDS-based $\mathrm{pH}_{\mathrm{e}}$ readout to map their therapeutic impact.

\section{Materials and Methods}

TmDOTP $^{5-}$ for BIRDS was purchased from Macrocyclics Inc. (Plano, TX, USA), while SPIO-NPs (Molday ION) were purchased from BioPAL Inc. (Worcester, MA, USA). The Molday ION (10 mg Fe/mL, dextran-coated, hydrodynamic diameter $30 \mathrm{~nm}$, zeta potential $-4.8 \mathrm{mV}$ ) were used without further modification or dilution to avoid altering their physical properties. Probenecid (used for temporary inhibition of renal clearance) was purchased from Sigma-Aldrich (St. Louis, MO, USA). Fischer 344 rats (male, 200-250 g) were obtained from Yale University vendors. RG2 and 9L tumor cell lines were purchased from American Type Culture Collections (Manassas, VA, USA). All animal experiments were conducted in accordance with Yale University's approved institutional animal care and use committee (IACUC) protocols. Tumor inoculation, animal preparation, and handling were conducted as described in our previous work $[57,58]$. In vivo magnetic resonance (MR) scans were conducted on 
a 9.4T Agilent (Santa Clara, CA, USA) or Bruker (Billerica, MA, USA) horizontal-bore spectrometer with a $1.4-\mathrm{cm}{ }^{1} \mathrm{H}$ surface RF coil.

2.1. Tumor Inoculation. The RG2 and 9L tumor cell lines were cultured and grown at $37^{\circ} \mathrm{C}$ and $5 \% \mathrm{CO}_{2}$ in DMEM media containing $10 \%$ heat-activated fetal bovine serum and $1 \%$ penicillin-streptomycin. The cells were harvested when they reached $80 \%$ confluence and suspended in serum-free media for inoculation. Rats were anesthetized with 3\% isoflurane and placed on a stereotactic holder. A heating pad was used to maintain the rat at physiological temperature $\left(36-37^{\circ} \mathrm{C}\right)$. An aliquot volume of $5 \mu \mathrm{L}$ with RG2 cells (1,250 cells) or 9L cells $(100,000$ cells) was injected into the right striatum $3 \mathrm{~mm}$ laterally to the right of bregma and $3 \mathrm{~mm}$ below the dura using a $10 \mu \mathrm{L}$ Hamilton syringe fitted with a 26gauge beveled needle. The $5 \mu \mathrm{L}$ volume was injected over the course of 5 minutes and the needle was left in place for an additional 5 minutes after the infusion stopped. The needle was then withdrawn slowly to prevent backflow of the cells. The cranial burr hole was sealed with bone wax. The scalp was sutured and treated with antibiotics to prevent infection. Meloxicam $(1 \mathrm{mg} / \mathrm{kg})$ was administered to prevent pain and inflammation.

2.2. Animal Preparation and Scanning. The tumor-bearing rats were scanned $\sim 3$ weeks after tumor inoculation when the tumor diameter was at least $\sim 3 \mathrm{~mm}$. The rats were anesthetized with $2 \%$ isoflurane, tracheotomized, and artificially ventilated $\left(70 \% \mathrm{~N}_{2} \mathrm{O} / 30 \% \mathrm{O}_{2}\right)$. The rats were placed on a heating pad to keep them warm during surgery. A femoral vein was cannulated with a PE-10 line for contrast agent administration $\left(1 \mathrm{mmol} / \mathrm{kg}\right.$ for $\mathrm{TmDOTP}^{5-}$ and $14 \mathrm{mg} \mathrm{Fe} / \mathrm{kg}$ for SPIO-NPs). A femoral artery was cannulated with a PE50 line for monitoring animal physiology $\left(\mathrm{pCO}_{2}, \mathrm{pO}_{2}, \mathrm{pH}\right.$, blood pressure) throughout the experiment. The rat was then anesthetized with $\alpha$-chloralose using an intraperitoneal line. To inhibit renal clearance and enhance contrast agent extravasation into the extracellular space and accumulation in the tumor, rats either received a coinfusion of TmDOTP ${ }^{5-}$ and probenecid $(n=5)$ or underwent renal ligation and infusion of $\operatorname{TmDOTP}^{5-}$ alone $(n=3)$. While renal ligation inhibits clearance efficiently, it is not suitable for longitudinal studies. Previously, we demonstrated that probenecid temporarily inhibited renal clearance when coinjected with the agent, thus enabling longitudinal studies and obviating the need for invasive renal surgeries [58]. Probenecid $(100 \mathrm{mg} / \mathrm{kg})$ was infused for 10 minutes $(24.5 \mu \mathrm{L} / \mathrm{min})$, followed by a waiting period of 20 minutes, and then coinfused slowly with TmDOTP ${ }^{5-}$ over a period of 90 minutes. A waterheating blanket was used to maintain body temperature of the animals between 36 and $37^{\circ} \mathrm{C}$ over the course of the experiment. A rectally placed fiber optic probe was used to monitor the body temperature during the scans.

2.3. MRI and BIRDS. In vivo transverse relaxation rate $\left(R_{2}\right)$ maps were obtained using a standard spin-echo sequence with 11 slices, $128 \times 128$ in-plane resolution, $1 \mathrm{~mm}$ slice thickness, field of view (FOV) $25 \times 25 \mathrm{~mm}^{2}$, recycle time (TR) $6 \mathrm{~s}$, and 12 different values of echo time (TE) from 10-120 ms. The transverse relaxivity $\left(r_{2}\right)$ of Molday ION (SPIO-NPs) was measured in vitro using the same pulse sequence using samples of varying concentrations of Molday ION $(1 \mathrm{mg} / \mathrm{kg}$ to $15 \mathrm{mg} / \mathrm{kg}$ ). The relaxivity was calculated from the slope of the linear fit of $R_{2}$ versus concentration. Although extreme $\mathrm{pH}$ changes can significantly alter properties of NPs, Liu et al. showed that the zeta potentials and hydrodynamic diameters of dextran-coated SPIO-NPs are fairly stable at physiologically relevant $\mathrm{pH}$ and ionic concentrations [63]. They showed that, between $\mathrm{pH} 4-8$ and media of different ionic strength $(0-140 \mathrm{mM})$, there was no aggregation of SPIO-NPs and that the change in hydrodynamic diameter of SPIO-NPs was less than $10 \mathrm{~nm}$, while the change in zeta potential was less than $10 \%$. Nevertheless, extreme $\mathrm{pH}$ changes could affect the physical features of SPIO-NPs. For example, a $\mathrm{pH}$ less than 4 could degrade the SPIO-NPs altogether, while a $\mathrm{pH}$ greater than 10 could lead to significant aggregation. Because we did not modify the SPIO-NPs or change their media and $\mathrm{pH}$, we do not expect property changes within the $\mathrm{pH}_{\mathrm{e}}$ range of tumors, normal tissue, and blood.

The rats were infused with $\mathrm{TmDOTP}^{5-}$ and the $3 \mathrm{D}$ CSI acquisition was started 40 minutes after TmDOTP $^{5-}$ infusion in both the probenecid coinfused and renal-ligated rats. TmDOTP ${ }^{5-}$ and similar lanthanide agents have previously been shown to cross the $\mathrm{BBB}$ and have been used to map whole brain $\mathrm{pH}_{\mathrm{e}}$ and temperature by BIRDS in healthy rodents $[60,64,65]$. We previously proposed that these agents slowly diffuse in the brain through the fenestrated vessels of circumventricular organs $[66,67]$. Moreover, diffusion of these agents from blood vessels into the extracellular space is enhanced by the high concentration gradient achieved by inhibition of renal clearance using renal ligation or coinfusion of the agent with probenecid $[58,60,64]$.

The 3D CSI datasets were acquired with a reduced spherical encoding of $\mathrm{k}$-space, as previously described [57], with a TR of $5 \mathrm{~ms}$, FOV of $25 \times 25 \times 25 \mathrm{~mm}^{3}$, and a nominal voxel resolution of $1 \mu \mathrm{L}$. A dual-banded refocused $90^{\circ}$ Shinnar-Le Roux (SLR) pulse of $35 \mathrm{kHz}$ bandwidth, $90 \mathrm{kHz}$ separation, and $205 \mu$ s duration was used to selectively excite the $\mathrm{H} 2 / \mathrm{H} 3$ and $\mathrm{H} 6$ protons of TmDOTP ${ }^{5-}$ (i.e., on either side of water). The phase encoded gradient duration was $160 \mu \mathrm{s}$, the spectral width was $250 \mathrm{kHz}$, and the acquisition time was $4.1 \mathrm{~ms}$. The total acquisition time for each 3D CSI dataset scan was 12 minutes. First a $\mathrm{pH}_{\mathrm{e}}$ map was acquired before the SPIO-NPs injection. Then a spin-echo dataset was obtained to determine the $R_{2}$ enhancement induced by TmDOTP ${ }^{5-}$. Next, the TmDOTP ${ }^{5-}$ infusion was stopped and SPIO-NPs were injected slowly (over 5 minutes). Then another spinecho dataset was obtained 15 minutes after the infusion of SPIO-NPs to determine the additional $R_{2}$ enhancement due to SPIO-NPs. Finally, infusion of remaining TmDOTP ${ }^{5-}$ dose was then resumed and another $\mathrm{pH}_{\mathrm{e}}$ map was obtained after infusion of SPIO-NPs.

$R_{2}$ maps were obtained by fitting the absolute MRI intensity at different TEs to a single exponential function using Matlab (Mathworks Inc., Natick, MA, USA). $R_{2}$ values from 
the 3 conditions (i.e., no contrast agent, after TmDOTP ${ }^{5-}$ infusion, and after SPIO-NPs infusion) were compared to determine the relaxation enhancement of each contrast agent. Average $R_{2}$ values were measured in regions of interest (ROIs), where $1 \mathrm{~mm}$ circular rings were taken from the center of mass of the tumor. The tumor edge was defined as regions $1 \mathrm{~mm}$ immediately outside the MRI-defined tumor core. Comparing the measured $R_{2}$ against the relaxivity of Molday ION allowed the amount of SPIO-NPs in each region to be approximated.

The 3D CSI datasets were used to create maps of the $\mathrm{H} 2, \mathrm{H} 3$, and $\mathrm{H} 6$ resonances of $\mathrm{TmDOTP}^{5-}$ before and after infusion of SPIO-NPs. The linewidth (LW) of the H6 resonance was measured to generate LW maps and create histograms before and after infusion of SPIO-NPs. While any of the three resonances could have been used to make the LW maps, H6 was chosen because it had the highest signalto-noise ratio (SNR). BIRDS-based $\mathrm{pH}_{\mathrm{e}}$ maps of the brain obtained with $\mathrm{TmDOTP}^{5-}$ were calculated as previously described $[57,58,60,62]$. Briefly, the 3D CSI datasets were reconstructed to a $25 \times 25 \times 25$ matrix using an in-house Matlab script. $\mathrm{pH}_{\mathrm{e}}$ was calculated by fitting the $\mathrm{H} 2, \mathrm{H} 3$, and H6 resonances (i.e., $\delta_{2}, \delta_{3}$, and $\delta_{6}$, respectively) to

$$
\mathrm{pH}_{\mathrm{e}}=a_{0}+\sum_{k=2,3,6} a_{1}^{k} \delta_{k}+\sum_{k=2,3,6} \sum_{j=2,3,6} a_{2}^{k j} \delta_{k} \delta_{j},
$$

where the coefficients $a_{0}, a_{1}^{k}$, and $a_{2}^{k j}$ were calculated from linear least-squares fit of $\mathrm{pH}_{\mathrm{e}}$ as a function of the resonances $\delta_{2}, \delta_{3}$, and $\delta_{6}[60]$. Average $\mathrm{pH}_{\mathrm{e}}$ values before and after infusion of SPIO-NPs were determined as a function of distance from the center of mass of the tumor, similar to the procedure described above for the $R_{2}$ maps.

2.4. Prussian Blue Iron Staining. Rats were sacrificed at the end of the experiments and brains were perfusion-fixed in $4 \%$ paraformaldehyde for Prussian blue iron staining to assess the distribution of SPIO-NPs. $10 \mu \mathrm{m}$ thick coronal sections of the fixed tissue were incubated in a solution of $4 \%$ potassium ferrocyanide and $4 \%$ hydrochloric acid twice for 10 minutes and then counterstained with nuclear fast red. Regions with $\mathrm{Fe}^{3+}$ (from SPIO-NPs) were expected to stain blue due to formation of ferric ferrocyanide.

\section{Results}

The $R_{2}$ maps before any contrast agent infusion (Figure 1(a)), after TmDOTP ${ }^{5-}$ infusion (Figure $1(\mathrm{~b})$ ), and after the infusion of SPIO-NPs (Figure 1(c)) are shown for a renal-ligated rat bearing an RG2 tumor. While tumor localization was obtained in all three MRI cases, much better delineation was observed upon enhancements by TmDOTP ${ }^{5-}$ alone or TmDOTP $^{5-}+$ SPIO-NPs. While $R_{2}$ increases were observed after infusion of $\mathrm{TmDOTP}^{5-}$ (relative to the intrinsic contrast), a superior MRI contrast was observed after the infusion of SPIO-NPs. The circular ROIs that were drawn from the tumor center are shown in Figure $1(\mathrm{~d})$. The $R_{2}$ relaxation enhancement was ROI-dependent with higher $R_{2}$ values inside the tumor and lower $R_{2}$ outside the tumor
(Figure 1(e)). Since $R_{2}$ enhancement was dependent on the concentration of the paramagnetic agents, the observed ROIspecific $R_{2}$ enhancement suggests that the extravasation (and accumulation) of both TmDOTP ${ }^{5-}$ and SPIO-NPs was highest in the tumor core and lower in regions farthest from the tumor's center of mass.

The $R_{2}$ values in the tumor (boundary marked by black outlines in Figures 1(a)-1(c)) were 22.5, 43.4, and $61.2 \mathrm{~s}^{-1}$ before contrast agent infusion, after infusion of $\mathrm{TmDOTP}^{5-}$, and after infusion of SPIO-NPs, respectively. For the healthy/nontumor tissue (contralateral side), the $R_{2}$ values were 25.8, 27.3, and $31.5 \mathrm{~s}^{-1}$ before contrast agent administration, after infusion of TmDOTP ${ }^{5-}$, and after infusion of SPIO-NPs, respectively. The measured $R_{2}$ relaxivity of Molday ION at $9.4 \mathrm{~T}$ in vitro was $2.45 \mathrm{~s}^{-1} \mathrm{mg}^{-1} \mathrm{Fe} / \mathrm{kg}$. By comparing the $R_{2}$ enhancement by SPIO-NPs against the relaxivity of the Molday ION, the average concentration of SPIO-NPs in the tumor (ROIs $1-3 \mathrm{~mm}$ ) was determined to be $7.27 \mathrm{mg} \mathrm{Fe} / \mathrm{kg}$. In healthy/nontumor tissue (ROIs 4-9 mm), there was a $4.1 \mathrm{~s}^{-1}$ change in $R_{2}$ with SPIO-NPs, which corresponds to $1.69 \mathrm{mg} \mathrm{Fe} / \mathrm{kg}$. Thus, the concentration of SPIO-NPs in the tumor was 4.3 times greater than in healthy/nontumor tissue, suggesting a fourfold enhanced extravasation/accumulation in the tumor.

Given the physical characteristics of Molday ION, we anticipate that the induced MRI effect is from SPIO-NPs within the extracellular milieu and calculating the concentration of SPIO-NPs in vivo should not be significantly affected by using the relaxivity measured in vitro. Girard et al. showed that the relaxivity of SPIO-NPs internalized in cells was lower than that of freely dispersed (in vitro) SPIO-NPs by as much as up to 4 times [68]. Taylor et al. also showed that the relaxivity of Molday ION internalized in cells was 4 times lower than the relaxivity in solution [69]. If we assume the relaxivity of Molday ION in vivo is 4 times lower than what was measured in vitro, then the calculated concentration of SPIO-NPs in both the tumor and healthy brain would be 4 times higher, but the relative distribution in tumor versus healthy/nontumor tissue would remain the same. For example, if we assume a $4 \mathrm{x}$ lower in vivo relaxivity $\left(0.61 \mathrm{mg}^{-1} \mathrm{~s}^{-1}\right.$ in vivo versus $2.45 \mathrm{mg}^{-1} \mathrm{~s}^{-1}$ in vitro), the concentration of SPIO-NPs in the tumor would be $29.16 \mathrm{mg} \mathrm{Fe} / \mathrm{kg}$ while the concentration in the healthy tissue would be $6.72 \mathrm{mg} \mathrm{Fe} / \mathrm{kg}$ (4.3 times lower than in the tumor). However, we expect that most of the SPIO-NPs will accumulate in the extracellular space where the microenvironment is more similar to the in vitro situation than that of SPIO-NPs internalized in cells. Moreover, we do not expect the relaxation of SPIO-NPs to change significantly over the $\mathrm{pH}$ range of our in vivo studies (i.e., $\mathrm{pH}_{\mathrm{e}} 6.8$ in tumors to 7.3 in healthy/nontumor tissue). Liu et al. and others have shown that the $R_{2}$ of dextrancoated SPIO-NPs was not significantly different over this $\mathrm{pH}$ range $[63,70]$. Using different concentrations of Molday ION, Shu et al. showed that the $R_{2}$ increase with increasing Molday ION dose was uniform across different brain regions [71]. Thus we expect the relaxivity of SPIO-NPs calculated in vitro to be a good approximation of the in vivo situation. Although we expect our concentration estimation to be 


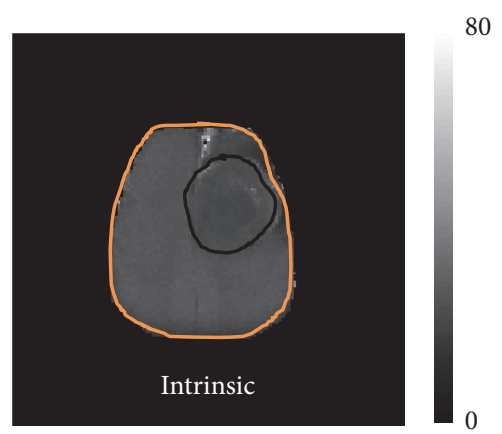

(a)

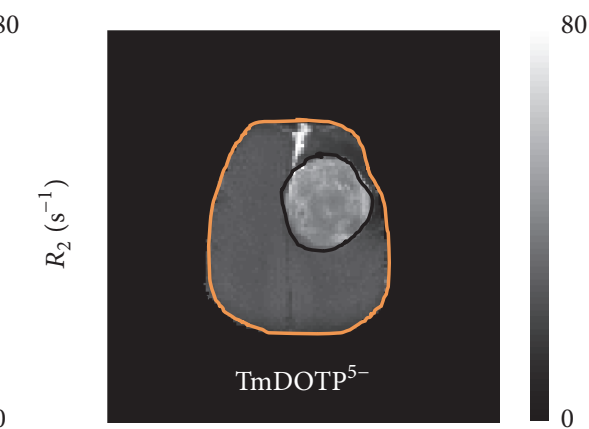

(b)

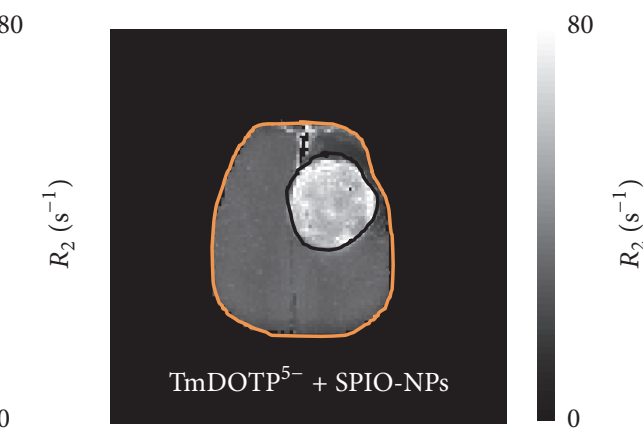

(c)

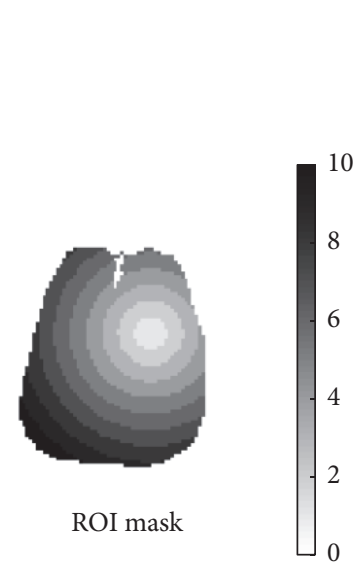

(d)

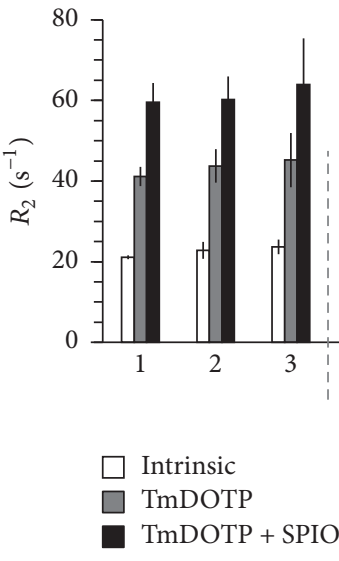

FIGURE 1: Transverse relaxation rate $\left(R_{2}\right)$ maps of an RG2 glioma-bearing rat that underwent renal ligation for TmDOTP ${ }^{5-}$ infusion, (a) without any contrast agent, (b) after infusion of TmDOTP ${ }^{5-}$, and (c) after infusion of SPIO-NPs. The scale bar in (a-c) denotes $R_{2}$ values from 0 to $80 \mathrm{~s}^{-1}$. Compared to the $R_{2}$ map before TmDOTP ${ }^{5-}$ infusion (a), the $R_{2}$ enhancement was observed throughout the brain after TmDOTP $^{5-}$ infusion (b), but superior $R_{2}$ enhancement and tumor delineation were observed following infusion of SPIO-NPs which also had cumulative effects from infusion of TmDOTP ${ }^{5-}$ (c). The contrast enhancement from both TmDOTP ${ }^{5-}$ and TmDOTP ${ }^{5-}$ with SPIO-NPs was region-specific, with highest enhancement in the tumor core and limited enhancement outside the tumor (relative to the intrinsic contrast, (a)). The black outline in $(\mathrm{a}-\mathrm{c})$ denotes the tumor boundary, which is based on the superior MRI contrast after infusion of SPIO-NPs. The region of interest (ROI) mask based on $1 \mathrm{~mm}$ circular rings from the tumor center $(\mathrm{d})$ was used to generate the radial $R_{2}$ distribution histogram of these ROIs (e). Scale bar in (d) denotes 0 to $10 \mathrm{~mm}$ diameter circular ROIs (portrayed on a representative rat brain slice). The gray dashed line in (e) denotes the demarcation between tumor and nontumor regions. The amount of SPIO-NPs in the tumor was 4.3 times greater than in the healthy tissue suggesting a preferential extravasation and accumulation of SPIO-NPs in the tumor. See Figure S1 for examples of Prussian blue staining for SPIO-NPs of an RG2 glioma-bearing rat that underwent renal ligation for TmDOTP ${ }^{5-}$ infusion. See Figure S2 for examples of $R_{2}$ maps of an RG2 glioma-bearing rat that underwent coinfusion of probenecid and TmDOTP ${ }^{5-}$.

only minimally affected, nevertheless these values should be considered "apparent" concentrations.

The preferential distribution of SPIO-NPs in tumors over healthy/nontumor tissues was tested with Prussian blue staining for $\mathrm{Fe}^{3+}$. Although the results from Prussian blue staining are not quantitative, regions that showed higher levels of SPIO-NPs were stained blue, indicating presence of $\mathrm{Fe}^{3+}$ (see Figure $\mathrm{S} 1$ in the Supplementary Material available online at https://doi.org/10.1155/2017/3849373). The Prussian blue stained images show an abundance of SPIO-NPs in the tumor, but very little staining on the healthy/nontumor contralateral side of the brain, supporting the enhanced accumulation of SPIO-NPs in the tumors observed with $R_{2}$ mapping.

Since the $R_{2}$ data shown in Figure 1 is from a renalligated rat, we obtained similar data from a rat that underwent coinfusion of $\mathrm{TmDOTP}^{5-}$ and probenecid (Figure S2). The amount of SPIO-NPs in the tumor was 2 times higher than in nontumor tissue (i.e., 4.5 versus $2.2 \mathrm{mg} \mathrm{Fe} / \mathrm{kg}$ ). In this case, the $R_{2}$ enhancement (Figure S2) was slightly lower than that observed in a renal-ligated rat (Figure 1), which is possibly due to higher TmDOTP ${ }^{5-} /$ SPIO-NPs concentration buildup in a renal-ligated rat.

Because acidic $\mathrm{pH}_{\mathrm{e}}$ is a hallmark of tumor pathology $[72,73]$, we obtained brain $\mathrm{pH}_{\mathrm{e}}$ maps in glioma-bearing rat brains with BIRDS using TmDOTP ${ }^{5-}$ before and after infusion of SPIO-NPs. We previously demonstrated that high concentrations of SPIO-NPs increase the LW of the $\mathrm{TmDOTP}^{5-}$ proton resonances in vitro [62]. In the current work, in addition to $\mathrm{pH}_{\mathrm{e}}$ maps, we also calculated the LW of the H6 proton of TmDOTP ${ }^{5-}$ in each voxel in the brain, before and after infusion of SPIO-NPs. Multimodal data 


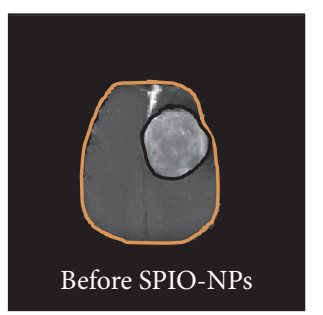

0 $R_{2}\left(\mathrm{~s}^{-1}\right)$

(i)

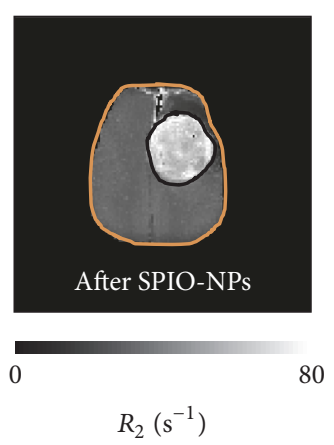

(i)

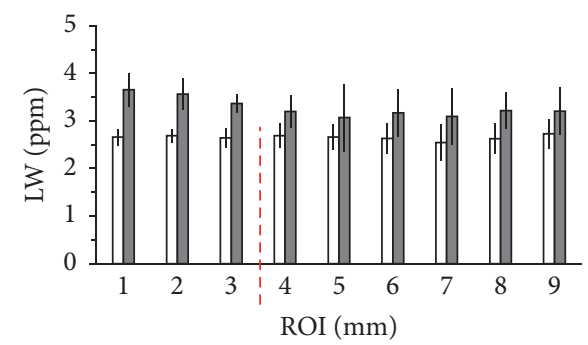

$\square$ Before SPIO

$\square$ After SPIO

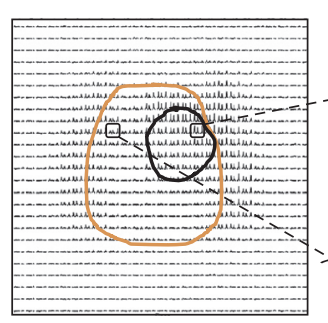

80 (1)

(ii)

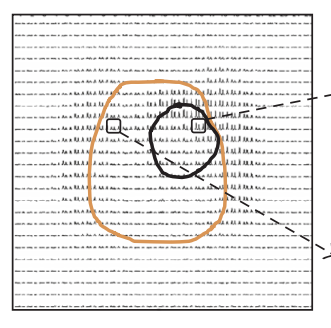

0

(ii)

(b)

(a)
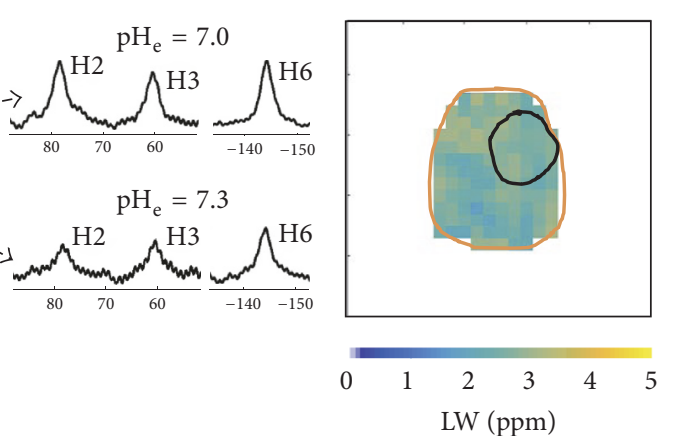

(iii)

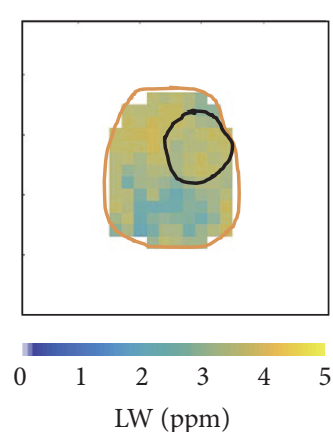

(iii)

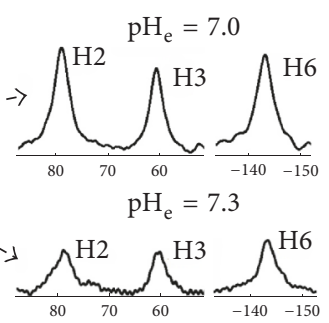

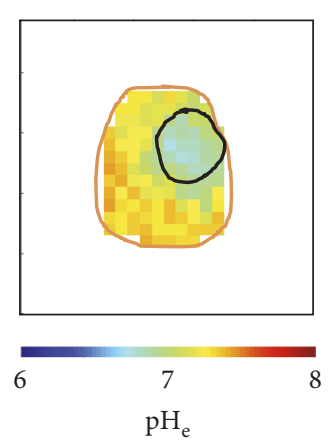

(iv)

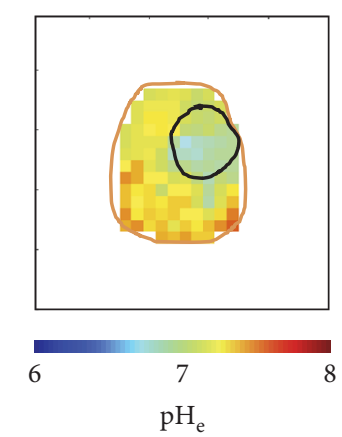

(iv)

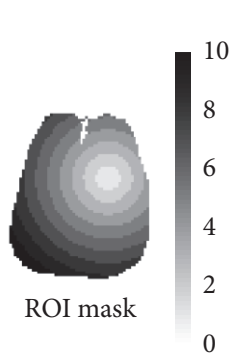

0

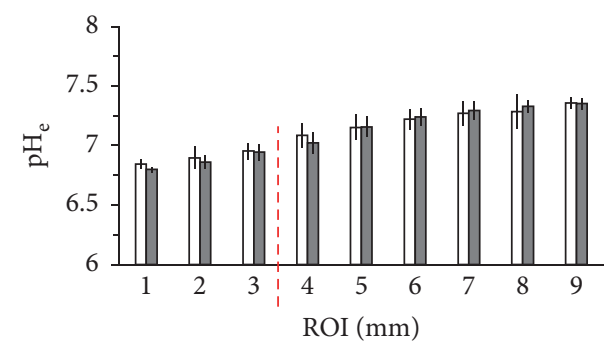

$\square$ Before SPIO
$\square$ After SPIO

(i)

(ii)

(c)

FIGURE 2: Multimodal data of relaxation rate $\left(R_{2}\right)$, chemical shift imaging (CSI), linewidth (LW), and extracellular $\mathrm{pH}$ ( $\left.\mathrm{pH}_{\mathrm{e}}\right)$ maps obtained for the same RG2 tumor-bearing rat in Figure 1, which had undergone renal ligation. ((a)(i)-(iv)) represent maps before the SPIO-NPs infusion while ((b)(i)-(iv)) represent the maps after the SPIO-NPs infusion. The $R_{2}$ maps were used to delineate and localize the tumor (black outline) and brain (orange outline) boundaries on the CSI, LW, and $\mathrm{pH}_{\mathrm{e}}$ maps. $R_{2}$ values inside the tumor increased significantly after infusion of SPIO-NPs. The CSI maps were used to create the $\mathrm{LW}$ maps and $\mathrm{pH}_{\mathrm{e}}$ maps. The LW increased after SPIO-NPs infusion especially in the tumor. The $\mathrm{pH}_{\mathrm{e}}$ values within the tumor core and also on the tumor margin were lower than in the healthy/nontumor regions. The panels between the CSI and the LW maps show examples of ${ }^{1} \mathrm{H}$ spectra of TmDOTP ${ }^{5-}$ protons from voxels inside and outside the tumor, revealing a significant intratumoral and peritumoral $\mathrm{LW}$ and $\mathrm{pH}_{\mathrm{e}}$ difference. A more detailed comparison was done using a region of interest (ROI) analysis of LW $((\mathrm{c})(\mathrm{i}))$ and $\mathrm{pH}_{\mathrm{e}}((\mathrm{c})(\mathrm{ii}))$ maps before and after the infusion of SPIO-NPs, using the ROI mask shown. The scale bar in the mask denotes 0 to $10 \mathrm{~mm}$ diameter circular ROIs (portrayed on a representative rat brain slice). The red dashed line denotes the demarcation between tumor (ROIs 1-3) and tumor edge (ROI 4)/nontumor regions (ROIs 5-9). See Figure S3 for an example of multimodal data of an RG2 tumor rat that underwent coinfusion of probenecid and $\mathrm{TmDOTP}^{5-}$.

( $R_{2}$ maps (i), CSI maps (ii), LW maps (iii), and $\mathrm{pH}_{\mathrm{e}}$ maps (iv)) before (Figure 2(a)) and after (Figure 2(b)) infusion of SPIO-NPs for the same RG2 tumor-bearing rat shown in Figure 1, which had undergone renal ligation, were obtained.
The $R_{2}$ maps (Figures 2(a)(i) and 2(b)(i)) were used to delineate and localize the tumor (black outline) and brain (orange outline) boundaries on the CSI, LW, and $\mathrm{pH}_{\mathrm{e}}$ maps. The CSI maps (Figures 2(a)(ii) and 2(b)(ii)) were used to 
TABLE 1: Regional analysis for relaxation rate $\left(R_{2}\right)$ for all RG2 tumor-bearing rats that underwent coinfusion of TmDOTP ${ }^{5-}$ and probenecid $(n=5)$. See Figure 3(a) for details. $R_{2}$ was measured inside the MRI-defined tumor core (see Figure S2), at the tumor edge (regions $1 \mathrm{~mm}$ outside the tumor boundary), and in the healthy/nontumor tissue before and after the infusion of SPIO-NPs. Data shown are mean and standard deviation (SD).

\begin{tabular}{|c|c|c|c|c|c|c|}
\hline \multirow{2}{*}{$R_{2}$} & \multicolumn{2}{|c|}{ Intrinsic } & \multicolumn{2}{|c|}{ TmDOTP $^{5-}$} & \multicolumn{2}{|c|}{$\mathrm{TmDOTP}^{5-}+$ SPIO-NPs } \\
\hline & Mean & SD & Mean & SD & Mean & SD \\
\hline Tumor core & 22.5 & 2.1 & 24.7 & 2.5 & 32.7 & 5.2 \\
\hline Tumor's edge & 22.7 & 4.0 & 23.7 & 4.4 & 28.6 & 7.9 \\
\hline Nontumor tissue & 23.8 & 3.8 & 23.2 & 3.4 & 27.3 & 3.7 \\
\hline
\end{tabular}

create the LW maps (Figures 2(a)(iii) and 2(b)(iii)) and $\mathrm{pH}_{\mathrm{e}}$ maps (Figures 2(a)(iv) and 2(b)(iv)). Examples of ${ }^{1} \mathrm{H}$ spectra of TmDOTP ${ }^{5-}$ protons from voxels inside and outside the tumor-illustrated in the panel between the CSI maps and LW maps-show that there is a significant intratumoral and peritumoral $\mathrm{LW}$ and $\mathrm{pH}_{\mathrm{e}}$ differences. The $\mathrm{SNR}$ was higher in the spectra after infusion of SPIO-NPs than before because TmDOTP $^{5-}$ infusion was resumed 15 minutes after the end of SPIO-NPs infusion. While the CSI maps (Figures 2(a)(ii) and 2(b)(ii)) show regionally varying $\mathrm{TmDOTP}^{5-}$ intensities, after infusion of SPIO-NPs, there is a clear variation in the LW maps, increasing from $\sim 2.5 \mathrm{ppm}$ globally before the infusion (Figure 2(a)(iii)) to $\sim 3.7 \mathrm{ppm}$ inside the tumor and $\sim 3.4 \mathrm{ppm}$ in the healthy/nontumor contralateral side of the brain (Figure 2(b)(iii)). A detailed ROI analysis of the average LWs shows similar LWs inside and outside the tumor before infusion of SPIO-NPs (Figure 2(c)(i), white bars). However, upon infusion of SPIO-NPs (Figure 2(c)(i), gray bars), the average LW increased (from 2.6 to $3.5 \mathrm{ppm}$ ) in the tumor (ROIs \# 1-3) and to a lesser extent (from 2.6 to $3.1 \mathrm{ppm}$ ) outside the tumor (ROIs \# 4-9). The LW broadening in the tumor correlated with the $R_{2}$ enhancement suggesting that the LW broadening was due to higher concentration of SPIONPs in the tumor.

The $\mathrm{pH}_{\mathrm{e}}$ maps (Figures 2(a)(iv) and 2(b)(iv)) were obtained by fitting the chemical shifts of the H2, H3, and H6 protons of TmDOTP ${ }^{5-}$ to equation (1) as previously described [60]. Although the CSI maps show regional variation of $\mathrm{TmDOTP}^{5-}$ proton intensities, both before and after infusion of SPIO-NPs (Figures 2(a)(ii) and 2(b)(ii)), the $\mathrm{pH}_{\mathrm{e}}$ calculation depends only on the chemical shifts of the nonexchangeable TmDOTP ${ }^{5-}$ protons and is independent of their concentration (or peak intensity) $[59,60]$. The $\mathrm{pH}_{\mathrm{e}}$ maps of RG2 tumors show lower $\mathrm{pH}_{\mathrm{e}}$ within the tumor core, but also beyond the tumor boundary, which is in good agreement with previous observations of this aggressive tumor type [57, 58]. Before injection of SPIO-NPs the average $\mathrm{pH}_{\mathrm{e}}$ was $7.0 \pm$ 0.1 within the tumor and $7.3 \pm 0.1$ in the healthy/nontumor tissue on the contralateral side for the RG2 tumor-bearing brain (Figure 2(a)(iv)). After injection of SPIO-NPs similar average $\mathrm{pH}_{e}$ values were observed in these regions (i.e., $7.0 \pm 0.1$ in the tumor and $7.3 \pm 0.1$ in healthy/nontumor tissue; Figure 2(b)(iv)). These in vivo results are consistent with our earlier in vitro report which showed that the $\mathrm{pH}$ readout and sensitivities of TmDOTP ${ }^{5-}$ are unaffected by the presence of paramagnetic agents like SPIO-NPs and $\mathrm{Gd}^{3+}$ agents [62]. Moreover, a detailed ROI analysis of the spatial $\mathrm{pH}_{\mathrm{e}}$ distribution shows that the average $\mathrm{pH}_{\mathrm{e}}$ increased as the $\mathrm{ROI}$ is positioned farther from the center of mass of the tumor (Figure 2(c)(ii)). However, no significant differences were observed between the average $\mathrm{pH}_{\mathrm{e}}$ values in each ROI before and after the SPIO-NPs infusion, indicating that BIRDSbased $\mathrm{pH}_{\mathrm{e}}$ mapping is not affected by the presence of SPIONPs.

Similar $R_{2}$, CSI, LW, and $\mathrm{pH}_{\mathrm{e}}$ maps as those shown in Figure 2 were obtained from rats, bearing RG2 tumors, that underwent coinfusion of TmDOTP ${ }^{5-}$ and probenecid (Figure S3). The results show that these distributions were similar to those observed in renal-ligated rats. Generally, the LWs increased after infusion of SPIO-NPs in all regions of the brain, but higher LW increases were observed inside the tumor. Before infusion of SPIO-NPs, the $\mathrm{pH}_{\mathrm{e}}$ was $6.85 \pm 0.03$ in the tumor and the $\mathrm{pH}_{\mathrm{e}}$ was $7.15 \pm 0.06$ in healthy/nontumor tissue (Figure S3 (A)(iv)). After infusion of SPIO-NPs, the $\mathrm{pH}_{\mathrm{e}}$ was $6.86 \pm 0.07$ in the tumor and the $\mathrm{pH}_{\mathrm{e}}$ was $7.17 \pm 0.06$ in healthy/nontumor tissue (Figure S3 (B)(iv)). The $\mathrm{pH}_{\mathrm{e}}$ of the tumor edge (ROI 4$)$ was also relatively acidified $(\mathrm{pH}$ $6.98 \pm 0.13$ before and $6.90 \pm 0.09$ after infusion of SPIONPs) compared to healthy/nontumor tissue farthest from the tumor core (ROIs 5-9).

Figure 3 shows the ROI analysis for $R_{2}$ and $\mathrm{pH}_{\mathrm{e}}$ before and after infusion of SPIO-NPs for all RG2 tumor-bearing rats that underwent coinfusion of TmDOTP ${ }^{5-}$ and probenecid ( $n=5)$. The $R_{2}$ enhancement was region-dependent (Figure 3(a); Table 1). Small $R_{2}$ enhancement was observed after infusion of TmDOTP ${ }^{5-}$, where $R_{2}$ increased by $2.2 \mathrm{~s}^{-1}$ in the tumor, $1.1 \mathrm{~s}^{-1}$ in the tumor edge, and no significant increase in the healthy/nontumor tissue. The tumor edge was defined as a circular ROI just $1 \mathrm{~mm}$ outside of the MRI defined tumor core. It is important to identify and analyze the tumor edge because after radiation therapy, it becomes edematous and harbors most therapy-resistant cells. Additionally, greater $R_{2}$ enhancement was observed upon infusion of SPIO-NPs (i.e., $R_{2}$ increase of $10.2 \mathrm{~s}^{-1}$ in tumor, $5.9 \mathrm{~s}^{-1}$ in tumor edge, and $4.1 \mathrm{~s}^{-1}$ in healthy/nontumor tissue). In contrast to the $R_{2}$ measurements, the average $\mathrm{pH}_{\mathrm{e}}$ values were not affected by the SPIO-NPs infusion (Figure 3(b); Table 2). However, $\mathrm{pH}_{\mathrm{e}}$ varied across regions; $\mathrm{pH}_{\mathrm{e}}$ was lowest $(6.9 \pm 0.1)$ in the tumor and highest $(7.2 \pm 0.1)$ in the healthy/nontumor tissue farthest from the tumor. Low $\mathrm{pH}_{\mathrm{e}}$ $(6.9 \pm 0.1)$ was also measured on the tumor edge. While the $\mathrm{pH}_{\mathrm{e}}$ of the tumor edge in RG2 gliomas was acidic 


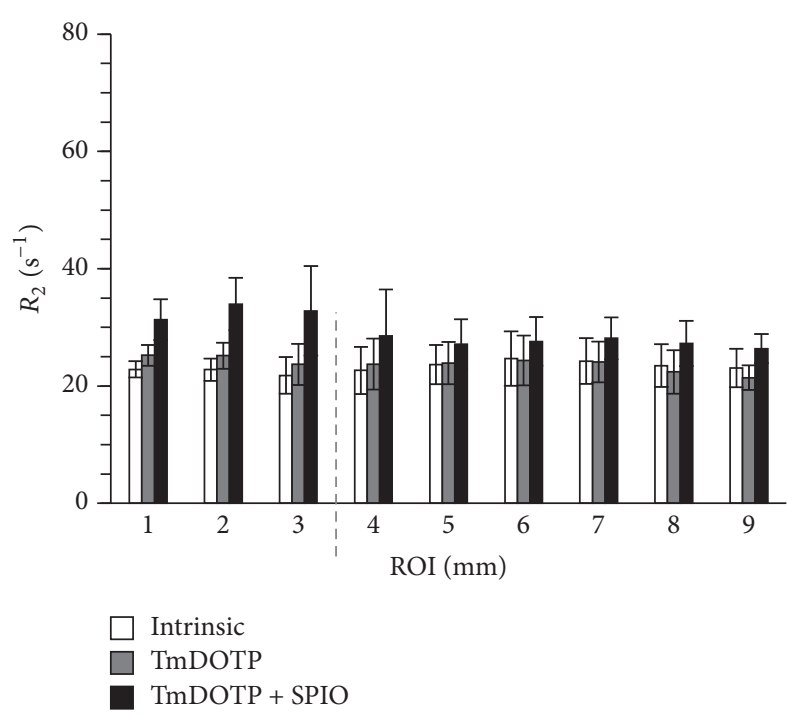

(a)

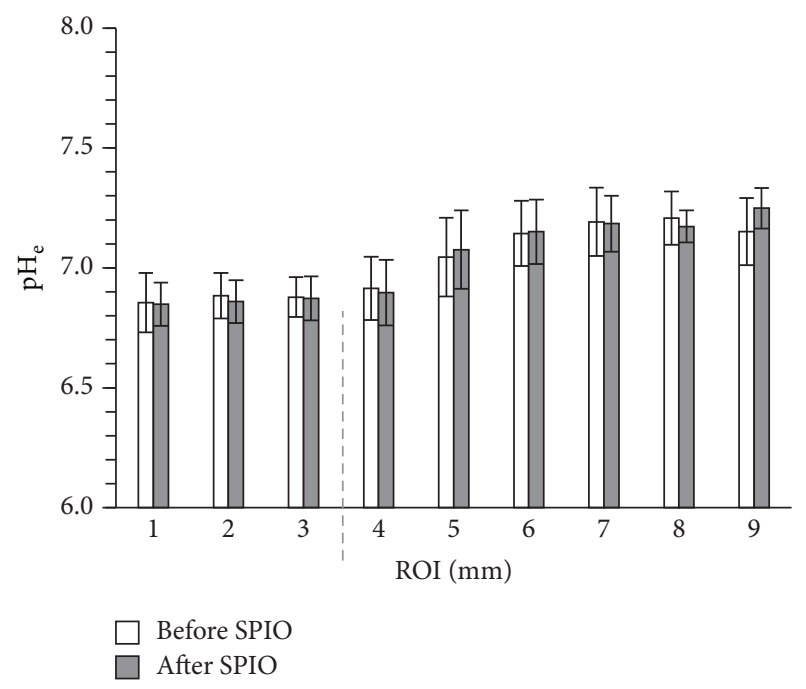

(b)

FIGURE 3: Region of interest (ROI) analysis for the average relaxation rate $\left(R_{2}\right)$ and extracellular $\mathrm{pH}\left(\mathrm{pH}_{\mathrm{e}}\right)$ before and after infusion of SPIONPs for all RG2 tumor-bearing rats that underwent coinfusion of $\operatorname{TmDOTP}^{5-}$ and probenecid $(n=5)$. The ROI analysis is based on concentric $1 \mathrm{~mm}$ circular rings drawn from the center of mass of the tumor. (a) Average $R_{2}$ values in different ROIs for intrinsic contrast, after infusion of TmDOTP ${ }^{5-}$, and after infusion of TmDOTP ${ }^{5-}$ with SPIO-NPs. The average $R_{2}$ enhancement was highest inside the tumor (ROIs 1-3) compared to tumor edge (ROI 4) and nontumor regions (ROIs 5-9). The dashed line represents the tumor edge. Small $R_{2}$ enhancement was observed after infusion of TmDOTP ${ }^{5-}$. However, much higher $R_{2}$ enhancement was observed upon infusion of SPIO-NPs. See Table 1 for details. (b) The average $\mathrm{pH}_{\mathrm{e}}$ values in different ROIs before and after infusion of SPIO-NPs. The $\mathrm{pH}_{\mathrm{e}}$ values measured before and after infusion of SPIO-NPs were similar, both inside and outside the tumor. The $\mathrm{pH}_{\mathrm{e}}$ was lowest in the tumor and highest in the healthy/nontumor tissue farthest from the tumor. Low $\mathrm{pH}_{\mathrm{e}}$ was also measured on the tumor margin. See Table 2 for details.

TABLE 2: Regional analysis for extracellular $\mathrm{pH}\left(\mathrm{pH}_{e}\right)$ imaging before and after infusion of SPIO-NPs for all RG2 tumor-bearing rats that underwent coinfusion of TmDOTP ${ }^{5-}$ and probenecid $(n=5)$. See Figure 3(b) for details. The $\mathrm{pH}_{\mathrm{e}}$ was measured inside the MRIdefined tumor core (see Figure S2), at the tumor edge (regions $1 \mathrm{~mm}$ outside the tumor boundary), and in the healthy/nontumor tissue before and after the infusion of SPIO-NPs. Data shown are mean and standard deviation (SD).

\begin{tabular}{lcccc}
\hline \multirow{2}{*}{$\mathrm{pH}_{\mathrm{e}}$} & \multicolumn{2}{c}{ Before SPIO-NPs } & \multicolumn{2}{c}{ After SPIO-NPs } \\
& Mean & SD & Mean & SD \\
\hline Tumor core & 6.9 & 0.1 & 6.9 & 0.1 \\
Tumor's edge & 6.9 & 0.1 & 6.9 & 0.1 \\
Nontumor tissue & 7.2 & 0.1 & 7.2 & 0.1 \\
\hline
\end{tabular}

relative to healthy/nontumor tissue, the $R_{2}$ enhancement between the tumor edge and the healthy/nontumor tissue were similar, suggesting that the vasculature in the tumor margin was still intact despite the acidic transformation of their microenvironment. Future experiments should look at the vascularization inside, around, and far beyond the tumor boundary, for example, with dynamic contrast enhanced MRI and with epidermal growth factor receptor staining.

In addition to measurements obtained in the aggressive RG2 glioma, we also acquired $\mathrm{pH}_{\mathrm{e}}$ maps before and after infusion of SPIO-NPs in rats bearing the less aggressive 9L gliosarcoma $(n=4)$ using coinfusion of $\operatorname{TmDOTP}^{5-}$ and probenecid (Figure 4). The $\mathrm{pH}_{\mathrm{e}}$ maps of the aggressive RG2 tumor (Figure 4(a)(i) before versus Figure 4(a)(ii) after infusion of SPIO-NPs) showed a lower $\mathrm{pH}_{\mathrm{e}}$ within the tumor region, but the acidification was diffuse and occurred also beyond the MRI-defined tumor boundary (see also Figures 2 and S3). Previously, it was reported that the diffuse acidification of $\mathrm{pH}_{\mathrm{e}}$ beyond the RG2 tumor boundary correlated with increased expression of the proliferation marker Ki-67 [57]. In contrast, the $\mathrm{pH}_{\mathrm{e}}$ maps of the less aggressive 9L gliosarcoma showed lower $\mathrm{pH}_{\mathrm{e}}$ only within the MRI-defined tumor core (Figure 4(b)(i) before versus Figure 4(b)(ii) after SPIONPs). A detailed ROI analysis of the $\mathrm{pH}_{e}$ maps shows that, for the $\mathrm{RG} 2$, the $\mathrm{pH}_{\mathrm{e}}$ slowly increases with the distance from the tumor core (Figure 4(a)(iii)), whereas for the 9L tumor the $\mathrm{pH}_{\mathrm{e}}$ is highest outside the tumor boundary and is distanceindependent (Figure 4(b)(iii)). Moreover, the regional $\mathrm{pH}_{\mathrm{e}}$ trends observed with BIRDS were not dependent on the type of infusion method, that is, coinfusion of $\mathrm{TmDOTP}^{5-}$ and probenecid versus infusion of $\mathrm{TmDOTP}^{5-}$ after renal ligation (Figure S4).

\section{Discussion}

Elevated aerobic glycolysis in gliomas leads to elevated lactic acid and proton production, which upon extrusion from the intracellular compartment results in acidification of the extracellular milieu [44]. Additionally, because the BBB is disrupted in gliomas, NPs loaded with imaging agents 


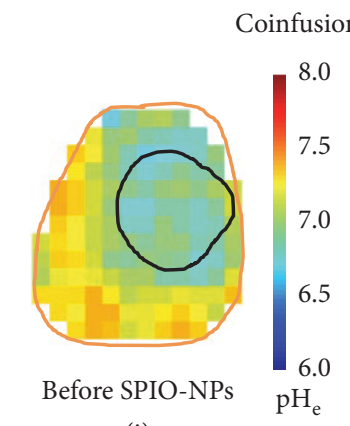

(i)

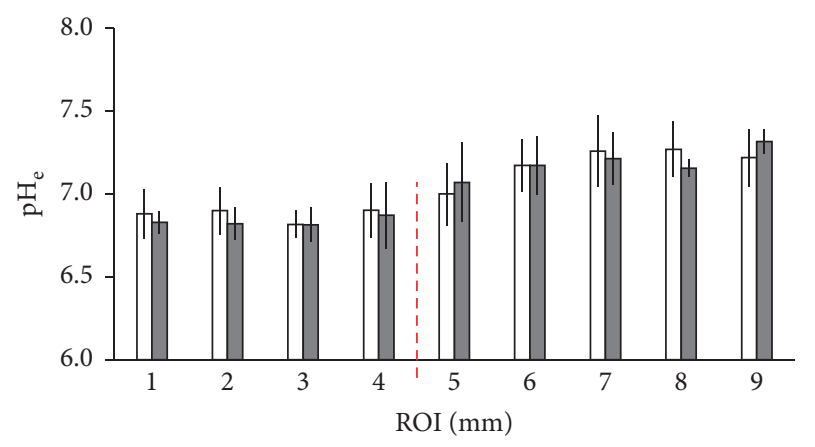

Before SPIO

After SPIO

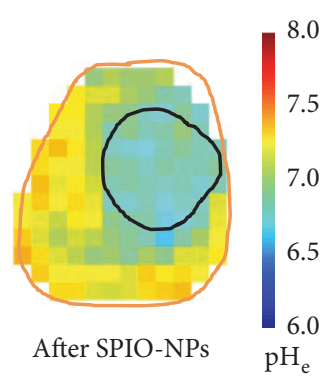

(ii)

Coinfusion (9L)

(i)

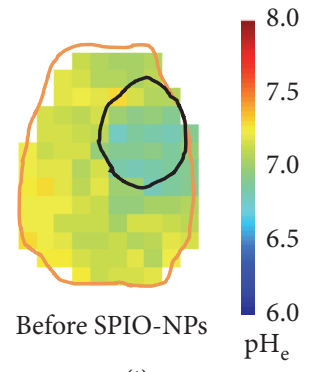

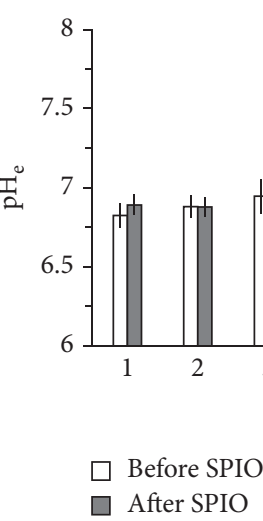

(iii)

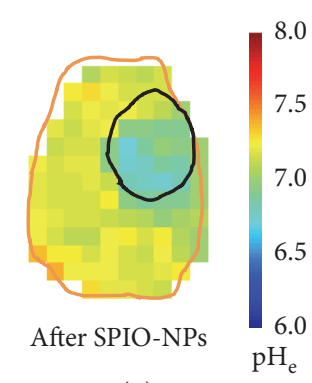

(ii)

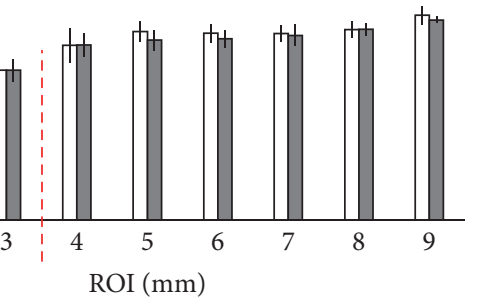

(iii)

(a)

(b)

FIGURE 4: Comparison of $\mathrm{pH}_{\mathrm{e}}$ maps for (a) RG2 glioma and (b) 9L gliosarcoma before and after infusion of SPIO-NPs in rats that underwent coinfusion of $\mathrm{TmDOTP}^{5-}$ and probenecid. In both (a) and (b), (i) and (ii) represent the $\mathrm{pH}_{\mathrm{e}}$ maps before and after SPIO-NPs infusion, respectively, while (iii) depicts a detailed ROI analysis. See Figure 1 for details of the ROI mask. In (a), the $\mathrm{pH}_{\mathrm{e}}$ inside the more aggressive RG2 glioma was typically lower than in the healthy/nontumor tissue, but diffuse acidification was observed well-beyond the MRI-defined tumor boundary. Thus the $\mathrm{pH}_{\mathrm{e}}$ slowly increased as the distance from the tumor core increased. In (b), the $\mathrm{pH}_{\mathrm{e}}$ inside the less aggressive 9L gliosarcoma was also lower than in the healthy/nontumor tissue, but the acidification did not extend beyond the MRI-defined tumor boundary, before and after infusion of SPIO-NPs. See Figure S4 for a comparison of regional $\mathrm{pH}_{\mathrm{e}}$ dependence on the method used for inhibition of renal clearance (renal ligation versus probenecid).

(e.g., SPIO-NPs) selectively permeate into and accumulate within tumors. In the present study, a region-specific $R_{2}$ enhancement from extravasation of SPIO-NPs was observed, with higher $R_{2}$ increases inside the tumor and smaller $R_{2}$ increases outside the tumor. Although SPIO-NPs affected MRI contrast in all tissues, excellent SPIO-induced MRI contrast delineated the glioma boundary due to greater extravasation of SPIO-NPs from the vasculature into the tumor relative to healthy/nontumor tissue. We also measured $\mathrm{pH}_{\mathrm{e}}$ with BIRDS using TmDOTP ${ }^{5-}$ before and after infusion of SPIO-NPs in rats bearing 9L and RG2 brain tumors. The results demonstrate that the $\mathrm{pH}_{\mathrm{e}}$ readout was unaffected by the presence of SPIO-NPs, because the intratumoralperitumoral $\mathrm{pH}_{\mathrm{e}}$ gradients were essentially identical before and after the infusion of SPIO-NPs, despite slight variations in LWs of the proton peaks for TmDOTP ${ }^{5-}$. The measured $\mathrm{pH}_{\mathrm{e}}$ was lowest inside the tumor and increased with the distance from the center of mass of the tumor in the more aggressive RG2 tumors. However, in the less aggressive 9L tumors, $\mathrm{pH}_{\mathrm{e}}$ was notably higher immediately outside the tumor boundary. We envisage coinjection of BIRDS agents (e.g., TmDOTP ${ }^{5-}$ ) and NPs containing drugs and SPIO, as a new methodology that can deliver high drug payloads to the tumor, image drug distribution, and track tumor location/size (by MRI), and at the same time monitor $\mathrm{pH}_{\mathrm{e}}$ response to therapy (by BIRDS) [74].

The brain's microvasculature is either degraded or immature in several neuropathologies, including glioblastomas. Breakthroughs in glioma imaging and therapy exploit the fact that NPs, containing either SPIO (for MRI) or drugs (for therapy), can extravasate through the leaky microvasculature. The SPIO-NPs extravasate into the tumor to generate superior MRI contrast while tumor-targeted D-NPs safely deliver high payloads of drugs to the tumor [74].

In the present study, the highest $R_{2}$ enhancement (from $\mathrm{TmDOTP}^{5-}$ and SPIO-NPs) occurred in the tumor and was lowest in healthy/nontumor tissue farthest from the tumor. Because the $R_{2}$ enhancement comes entirely from the infused agents, this region-specific enhancement suggests a corresponding spatial variation in vascular permeability and consequent extravasation. In addition to the enhanced extravasation, the chaotic vascular architecture in tumors 
contributes to poor clearance leading to increased retention of SPIO-NPs in the interstitial space of the tumor core. By using the $R_{2}$ enhancement and the relaxivity of Molday ION (SPIO-NPs), we calculated that the amount of SPIO-NPs in the tumor was 2 to 4 times higher than in healthy/nontumor tissue. The EPR in tumors has been widely utilized to preferentially deliver high amounts of imaging agents and DNPs, both passively and actively [75, 76].

High-grade solid brain tumors tend to develop necrotic cores due to a combination of poor vascularization and inadequate perfusion [77-79]. Because gliomas like RG2 are very aggressive, they rapidly invade to induce severe neurological problems. As a consequence the rodent reaches terminal situations before the tumor cores are able to become necrotic. For example, these rodent brain tumors grow within a few weeks, whereas in the human brain gliomas develop necrotic foci after many months, if not longer. Tumor necrosis has very likely not yet occurred in these rodent tumors at the time points of our experiments. The observed higher $R_{2}$ in the center relative to the periphery suggests higher permeation and accumulation of SPIO-NPs in the center of the tumor due to greater extent of $\mathrm{BBB}$ disruption within the tumor niche. Prior studies support these observations. Beaumont et al. did not observe any necrosis in their RG2 rat gliomas at similar time points as our experiments [16]. Their staining results also showed that the BBB was significantly disrupted at the center of the tumor in RG2 tumors. While the vasculature at the primary tumor site/core is leaky, the blood vessels at the tumor infiltration sites (i.e., periphery) are often immature, which may slow the extravasation of SPIO-NPs out of the blood into these new tumor sites. Uehara et al. also showed that necrosis of tumor cores is minimal or absent in RG2 tumors at time points less than 4 weeks following inoculation [80]. Therefore based on the information regarding RG2 tumor growth from prior work in this and other laboratories, we expect the tumor cores to be non-necrotic, and thus higher $R_{2}$ increase from the SPIO-NPs would be observed in the tumor core. Additionally, because gliomas including RG2 are known to have an increased presence of macrophages relative to healthy brain tissue, the higher amount of SPIO-NPs in the tumor could be due in part to macrophage phagocytosis [16].

4.1. Superparamagnetic Iron Oxide Nanoparticles in Cancer Theranostics. Owing to their strong superparamagnetic properties, tunable size, shape, coating, and magnetic susceptibility, SPIO-NPs have gained utility as therapeutic agents in alternating magnetic field hyperthermia [81-85], as MRI contrast agents for cell tracking [86-89], and for imaging tumor location/size as well as drug delivery [40, $74,90]$. Drug delivery imaging with SPIO-NPs is often accomplished by coencapsulating drugs and SPIO into a given nanocarrier platform (e.g., micelles or liposomes). In liposomes, for example, SPIO-NPs and hydrophilic drugs can be encapsulated inside the nanocarrier, whereas hydrophobic drugs can be incorporated on the nanocarrier membrane. Recent advances also involve coating the surface of SPIONPs itself with drugs [11, 12]. Entry and accumulation of these drug-containing and SPIO-containing NPs into the tumor have been achieved by passive targeting, whereby the
NPs are small enough to extravasate through leaky tumor vasculature, but large enough not to cross the intact vessels in healthy/nontumor tissue. However, better and more selective targeting is achieved when the NPs are coated with ligands that are specific to receptors and/or transporters that are overexpressed on tumor cells and vasculature. Examples of such targets include transferrin receptors, epidermal growth factor receptors, folate receptors, vascular endothelial growth factor receptors, monocarboxylate transporters, and glucose transporters [29, 91-96]. In all these cases, the delivery and biodistribution of D-NPs are visualized and quantified through signal attenuation (negative contrast) of the $R_{2}$ weighted MRI resulting from the strong superparamagnetic fields generated by SPIO-NPs. Because both the drugs and SPIO-NPs are contained in the same nanocarrier, the location and distribution of the SPIO-NPs, as observed by MRI, reflect the biodistribution of D-NPs. By quantifying the SPIOinduced MRI contrast attenuation, it is possible to quantify the D-NPs delivered to the tumor.

Currently, measurement of tumor size is the only FDAapproved method to assess the response to therapy noninvasively. Because changes in tumor size following treatment may take up to a month to manifest, this method is not ideal for aggressive brain cancers, especially when the treatment is later found not to have been effective. Thus a clear need exists for methods that can provide prompt assessment of therapeutic efficacy so that treatment can be altered quickly if desired. Recently, it was shown that quantitative monitoring of the tumor microenvironment following a pharmacologic challenge provides a better way to monitor therapeutic efficacy [97]. Because acidification of $\mathrm{pH}_{\mathrm{e}}$ promotes drug resistance, degradation of the extracellular matrix, angiogenesis, tumor invasion, and metastasis, drugs that raise (or neutralize) $\mathrm{pH}_{\mathrm{e}}$ by targeting the acid-generating glycolysis in tumors have demonstrated significant inhibition of tumor growth and enhanced apoptosis [45, 46, 48, 72, 98, 99]. Additionally, drugs that directly raise tumor $\mathrm{pH}_{\mathrm{e}}$ (e.g., bicarbonate treatment) inhibit tumor invasion and metastasis [100, 101]. Because bicarbonate and drugs that inhibit glycolysis elevate $\mathrm{pH}_{\mathrm{e}}$ in a few days, methods that quantitatively measure tumor $\mathrm{pH}_{\mathrm{e}}$ longitudinally may provide an effective evaluation of their therapeutic efficacy and allow for prompt modification of therapy if the initial treatment is not working. A recent study has reported that temozolomide, which is an alkylating agent and is adjuvant chemotherapy used to clinically treat glioblastomas, arrests glioma growth and normalizes intratumoral $\mathrm{pH}_{\mathrm{e}}$ [102].

4.2. Combining Drug Delivery Imaging with $\mathrm{pH}_{e}$ Imaging to Assess Therapy. Given the significant relaxation enhancement of the nonexchangeable protons on the TmDOTP ${ }^{5-}$ agent $[60,103,104]$ due to pseudocontact interactions with unpaired $\mathrm{Tm}^{3+}$ electrons, we hypothesized that BIRDS-based $\mathrm{pH}_{\mathrm{e}}$ readout of $\mathrm{TmDOTP}^{5-}$ will remain uncompromised by SPIO-NPs. Although SPIO-NPs altered MRI contrast in all tissues, SPIO-based MRI contrast clearly demarcated the tumor boundary due to greater extravasation of NPs through leaky blood vessels. Nonetheless, the quality of BIRDS-based 
$\mathrm{pH}_{\mathrm{e}}$ readout with $\mathrm{TmDOTP}^{5-}$, for both intratumoral and peritumoral regions, was unaffected by the presence of the SPIO-NPs, since the $\mathrm{pH}_{\mathrm{e}}$ maps obtained before and after the infusion of SPIO-NPs were very similar.

While separate infusions of TmDOTP ${ }^{5-}$ and SPIO-NPs were employed in the present study, future studies might assess the possibility of combining them [74]. Conjugating several monomers of the $\mathrm{pH}_{\mathrm{e}}$-sensitive agent on the surface of the NPs could possibly enhance the sensitivity of BIRDS to monitor the immediate environment of D-NPs and prolong their lifetime to enable multiple monitoring sessions at various treatment time points. Ordinarily, BIRDS agents have fast renal clearance owing to their small size and thus renal inhibition is necessary for accumulation $[57,58$, $60,105]$. However, if conjugated to NPs, the BIRDS agents lifetime might increase significantly (i.e., to several days, which is the case for SPIO-NPs), thus allowing their use without inhibition of renal clearance and obviating the need for repeated infusions [106]. Towards this goal, it has been previously demonstrated in vitro that encapsulation of BIRDS agents in liposomal nanoparticles resulted in an MR signal amplification without impeding the local $\mathrm{pH}$ readout [62].

\section{Summary}

The treatment of brain gliomas is hampered in part by a limited availability of reliable in vivo methodologies that can simultaneously and noninvasively measure glioma invasion, drug delivery, and its therapeutic benefits. In this study, we demonstrated superb MRI contrast enhancement and tumor delineation with SPIO-NPs and quantitative imaging of intratumoral-peritumoral $\mathrm{pH}_{\mathrm{e}}$ gradients using BIRDS in rat models of brain gliomas. Furthermore, we demonstrated that both the intratumoral and peritumoral $\mathrm{pH}_{\mathrm{e}}$ readouts, measured with BIRDS using TmDOTP ${ }^{5-}$, are not compromised by the presence of SPIO-NPs. Thus, we propose a new cancer imaging protocol that can target high drug payloads (via DNPs) to tumors and image the drug delivery (via SPIO-NPs), concurrently map tumor location and size (by MRI), and at the same time monitor therapeutic efficacy through druginduced changes in $\mathrm{pH}_{\mathrm{e}}$ (by BIRDS) [74].

\section{Abbreviations}

3-APP: 3-Aminopropyl phosphonate

BBB: Blood brain barrier

BIRDS: Biosensor imaging of redundant deviation in shifts

CEST: Chemical exchange saturation transfer

CSI: Chemical shift imaging

EPR: Enhanced permeation and retention

FOV: Field of view

LW: Linewidth

MR: Magnetic resonance

NPs: Nanoparticles

$\mathrm{pH}_{\mathrm{e}}$ : Extracellular $\mathrm{pH}$

ROI: Region of interest

SD: $\quad$ Standard deviation

\author{
SNR: $\quad$ Signal-to-noise ratio \\ SLR: $\quad$ Shinnar-Le Roux \\ SPIO: $\quad$ Superparamagnetic iron oxide \\ TmDOTP $^{5-}$ : Thulium 1,4,7,10-tetraazacyclododecane- \\ 1,4,7,10-tetrakis methylene \\ phosphonate.
}

\section{Disclosure}

Parts of this work have previously been presented as a published abstract in BRAIN \& BRAIN PET 2017 Poster Viewing Session III.

\section{Conflicts of Interest}

The authors declare no conflicts of interest.

\section{Authors' Contributions}

Samuel Maritim and Fahmeed Hyder designed research. Samuel Maritim, Daniel Coman, Yuegao Huang, Jyotsna U. Rao, John J. Walsh, and Fahmeed Hyder performed research. Samuel Maritim, Daniel Coman, Yuegao Huang, Jyotsna U. Rao, and Fahmeed Hyder analyzed data. Samuel Maritim, Daniel Coman, Yuegao Huang, Jyotsna U. Rao, and Fahmeed Hyder wrote the paper.

\section{Acknowledgments}

This paper is supported by NIH Grants (R01 EB-023366, R01 EB-011968, and R01 CA-140102).

\section{References}

[1] J. Ferlay, H. R. Shin, F. Bray, D. Forman, C. Mathers, and D. M. Parkin, "Estimates of worldwide burden of cancer in 2008: GLOBOCAN 2008," International Journal of Cancer, vol. 127, no. 12, pp. 2893-2917, 2010.

[2] H. Ohgaki, "Epidemiology of brain tumors," Methods in Molecular Biology, vol. 472, pp. 323-342, 2009.

[3] H. F. Bradford, "Glutamate, GABA and epilepsy," Progress in Neurobiology, vol. 47, no. 6, pp. 477-511, 1995.

[4] W. M. Pardridge, "Drug targeting to the brain," Pharmaceutical Research, vol. 24, no. 9, pp. 1733-1744, 2007.

[5] W. M. Pardridge, "Blood-brain barrier delivery," Drug Discovery Therapy, vol. 12, no. 1-2, pp. 54-61, 2007.

[6] H. Wolburg and A. Lippoldt, "Tight junctions of the bloodbrain barrier: development, composition and regulation," Vascul Pharmacol, vol. 38, no. 6, pp. 323-337, 2002.

[7] M. Demeule, A. Regina, J. Jodoin et al., "Drug transport to the brain: Key roles for the efflux pump P-glycoprotein in the bloodbrain barrier," Vascular Pharmacology, vol. 38, no. 6, pp. 339$348,2002$.

[8] W. Risau and H. Wolburg, "Development of the blood-brain barrier," Trends in Neurosciences, vol. 13, no. 5, pp. 174-178, 1990.

[9] S.-W. Lee, J. K. Woo, J. A. Park, K. C. Yoon, Y.-W. Kwon, and K.-W. Kim, "Blood-brain barrier interfaces and brain tumors," Archives of Pharmacal Research, vol. 29, no. 4, pp. 265-275, 2006. 
[10] J. D. Huber, R. D. Egleton, and T. P. Davis, "Molecular physiology and pathophysiology of tight junctions in the blood -brain barrier," Trends in Neurosciences, vol. 24, no. 12, pp. 719-725, 2001.

[11] P.-C. Liang, Y.-C. Chen, C.-F. Chiang et al., "Doxorubicinmodified magnetic nanoparticles as a drug delivery system for magnetic resonance imaging-monitoring magnet-enhancing tumor chemotherapy," International Journal of Nanomedicine, vol. 11, pp. 2021-2037, 2016.

[12] Y. Ling, K. Wei, F. Zou, and S. Zhong, “Temozolomide loaded PLGA-based superparamagnetic nanoparticles for magnetic resonance imaging and treatment of malignant glioma," International Journal of Pharmaceutics, vol. 430, no. 1-2, pp. 266-275, 2012.

[13] H. Sarin, "Physiologic upper limits of pore size of different blood capillary types and another perspective on the dual pore theory of microvascular permeability," Journal of Angiogenesis Research, vol. 2, no. 1, article no. 14, 2010.

[14] D. J. Cox, G. J. Pilkington, and P. L. Lantos, “The fine structure of blood vessels in ethylnitrosourea-induced tumours of the rat nervous system: with special reference to the breakdown of the blood-brain barrier," Journal of Experimental Pathology, vol. 57, pp. 419-430, 1976.

[15] K. E. Schlageter, P. Molnar, G. D. Lapin, and D. R. Groothuis, "Microvessel organization and structure in experimental brain tumors: Microvessel populations with distinctive structural and functional properties," Microvascular Research, vol. 58, no. 3, pp. 312-328, 1999.

[16] M. Beaumont, B. Lemasson, R. Farion, C. Segebarth, C. Rémy, and E. L. Barbier, "Characterization of tumor angiogenesis in rat brain using iron-based vessel size index MRI in combination with gadolinium-based dynamic contrast-enhanced MRI," Journal of Cerebral Blood Flow \& Metabolism, vol. 29, no. 10, pp. 1714-1726, 2009.

[17] D. Feng, J. A. Nagy, A. M. Dvorak, and H. F. Dvorak, "Different pathways of macromolecule extravasation from hyperpermeable tumor vessels," Microvascular Research, vol. 59, no. 1, pp. 24-37, 2000.

[18] D. Feng, J. A. Nagy, H. F. Dvorak, and A. M. Dvorak, "Ultrastructural studies define soluble macromolecular, particulate, and cellular transendothelial cell pathways in venules, lymphatic vessels, and tumor-associated microvessels in man and animals," Microscopy Research and Technique, vol. 57, no. 5, pp. 289-326, 2002.

[19] H. Hashizume, P. Baluk, S. Morikawa et al., "Openings between defective endothelial cells explain tumor vessel leakiness," The American Journal of Pathology, vol. 156, no. 4, pp. 1363-1380, 2000.

[20] N. A. Vick and D. D. Bigner, "Microvascular abnormalities in virally-induced canine brain tumors. Structural bases for altered blood-brain barrier function," Journal of the Neurological Sciences, vol. 17, no. 1, pp. 29-39, 1972.

[21] R. G. Blasberg and D. R. Groothuis, "Chemotherapy of BrainTumors - Physiological and Pharmacokinetic Considerations," Seminars in Oncology, vol. 13, pp. 70-82, 1986.

[22] D. R. Groothuis, J. M. Fischer, G. Lapin, N. A. Vick, and D. D. Bigner, "Comparative Permeability of Different Glioma Models to Horseradish-Peroxidase," Cancer Treat Rep, vol. 65, pp. 13-18, 1982.

[23] C. H. Tator, "Retention of tritiated methotrexate in a transplantable mouse glioma," Cancer Research, vol. 36, pp. 30583066, 1976.
[24] W. L. Monsky, C. Mouta Carreira, Y. Tsuzuki, T. Gohongi, D. Fukumura, and R. K. Jain, "Role of host microenvironment in angiogenesis and microvascular functions in human breast cancer xenografts: mammary fat pad versus cranial tumors," Clinical cancer research : an official journal of the American Association for Cancer Research, vol. 8, pp. 1008-1013, 2002.

[25] F. Yuan, H. A. Salehi, Y. Boucher, U. S. Vasthare, R. F. Tuma, and R. K. Jain, "Vascular permeability and microcirculation of gliomas and mammary carcinomas transplanted in rat and mouse cranial windows," Cancer Research, vol. 54, pp. 45644568,1994

[26] A. G. De Boer and P. J. Gaillard, "Strategies to improve drug delivery across the blood-brain barrier," Clinical Pharmacokinetics, vol. 46, no. 7, pp. 553-576, 2007.

[27] A. G. de Boer and P. J. Gaillard, "Drug targeting to the brain," Annual Review of Pharmacology and Toxicology, vol. 47, pp. 323355, 2007.

[28] F. M. Kievit, O. Veiseh, C. Fang et al., "Chlorotoxin labeled magnetic nanovectors for targeted gene delivery to glioma," ACS Nano, vol. 4, no. 8, pp. 4587-4594, 2010.

[29] S. D. Weitman, K. M. Frazier, and B. A. Kamen, "The folate receptor in central nervous system malignancies of childhood," Journal of Neuro-Oncology, vol. 21, no. 2, pp. 107-112, 1994.

[30] S. P. S. Howarth, T. Y. Tang, R. Trivedi et al., "Utility of USPIOenhanced MR imaging to identify inflammation and the fibrous cap: A comparison of symptomatic and asymptomatic individuals," European Journal of Radiology, vol. 70, no. 3, pp. 555-560, 2009.

[31] K. Motomura, M. Ishitobi, Y. Komoike et al., "SPIO-enhanced magnetic resonance imaging for the detection of metastases in sentinel nodes localized by computed tomography lymphography in patients with breast cancer," Annals of Surgical Oncology, vol. 18, no. 12, pp. 3422-3429, 2011.

[32] K. Polakova, I. Mocikova, D. Purova et al., "Magnetic resonance cholangiopancreatography (MRCP) using new negative peroral contrast agent based on superparamagnetic iron oxide nanoparticles for extrahepatic biliary duct visualization in liver cirrhosis," Biomedical Papers, vol. 160, no. 4, pp. 512-517, 2016.

[33] M. Sigovan, W. Gasper, H. F. Alley, C. D. Owens, and D. Saloner, "USPIO-enhanced MR angiography of arteriovenous fistulas in patients with renal failure," Radiology, vol. 265, no. 2, pp. 584590, 2012.

[34] T. Tang, S. P. S. Howarth, S. R. Miller et al., "Assessment of inflammatory burden contralateral to the symptomatic carotid stenosis using high-resolution ultrasmall, superparamagnetic iron oxide-enhanced MRI," Stroke, vol. 37, no. 9, pp. 2266-2270, 2006.

[35] T. Y. Tang, A. J. Patterson, S. R. Miller et al., "Temporal dependence of in vivo USPIO-enhanced MRI signal changes in human carotid atheromatous plaques," Neuroradiology, vol. 51, no. 7, pp. 457-465, 2009.

[36] T. Tourdias, S. Roggerone, M. Filippi et al., "Assessment of disease activity in multiple sclerosis phenotypes with combined gadolinium- and superparamagnetic iron oxide-enhanced MR imaging," Radiology, vol. 264, no. 1, pp. 225-233, 2012.

[37] M. Wagner, S. Wagner, J. Schnorr et al., "Coronary MR angiography using citrate-coated very small superparamagnetic iron oxide particles as blood-pool contrast agent: Initial experience in humans," Journal of Magnetic Resonance Imaging, vol. 34, no. 4, pp. 816-823, 2011. 
[38] C. Sun, O. Veiseh, J. Gunn et al., "In vivo MRI detection of gliomas by chlorotoxin-conjugated superparamagnetic nanoprobes," Small, vol. 4, no. 3, pp. 372-379, 2008.

[39] C. Sun, C. Fang, Z. Stephen et al., "Tumor-targeted drug delivery and MRI contrast enhancement by chlorotoxin-conjugated iron oxide nanoparticles," Nanomedicine, vol. 3, no. 4, pp. 495$505,2008$.

[40] G. Strohbehn, D. Coman, L. Han et al., "Imaging the delivery of brain-penetrating PLGA nanoparticles in the brain using magnetic resonance," Journal of Neuro-Oncology, vol. 121, no. 3, pp. 441-449, 2015.

[41] W. Li, Z. Zhang, A. C. Gordon et al., "SPIO-labeled yttrium microspheres for MR imaging quantification of transcatheter intrahepatic delivery in a rodent model," Radiology, vol. 278, no. 2, pp. 405-412, 2016.

[42] M. Stubbs, P. M. J. McSheehy, J. R. Griffiths, and C. L. Bashford, "Causes and consequences of tumour acidity and implications for treatment," Molecular Medicine Today, vol. 6, no. 1, pp. 15-19, 2000.

[43] R. A. Cardone, V. Casavola, and S. J. Reshkin, "The role of disturbed $\mathrm{pH}$ dynamics and the $\mathrm{NA}+/ \mathrm{H}+$ exchanger in metastasis," Nature Reviews Cancer, vol. 5, no. 10, pp. 786-795, 2005.

[44] R. A. Gatenby, E. T. Gawlinski, A. F. Gmitro, B. Kaylor, and R. J. Gillies, "Acid-mediated tumor invasion: a multidisciplinary study," Cancer Research, vol. 66, no. 10, pp. 5216-5223, 2006.

[45] A. I. Hashim, X. Zhang, J. W. Wojtkowiak, G. V. Martinez, and R. J. Gillies, "Imaging $\mathrm{pH}$ and metastasis," NMR in Biomedicine, vol. 24 , no. 6 , pp. 582-591, 2011.

[46] R. Martinez-Zaguilan, E. A. Seftor, R. E. Seftor, Y. W. Chu, R. J. Gillies, and M. J. Hendrix, "Acidic $\mathrm{pH}$ enhances the invasive behavior of human melanoma cells," Clinical \& Experimental Metastasis, vol. 14, pp. 176-186, 1996.

[47] R. A. Gatenby and R. J. Gillies, "Why do cancers have high aerobic glycolysis?” Nature Reviews Cancer, vol. 4, no. 11, pp. 891-899, 2004.

[48] J. Barar and Y. Omidi, "Dysregulated $\mathrm{pH}$ in tumor microenvironment checkmates cancer therapy," Bioimpacts, vol. 3, no. 4, pp. 149-162, 2013.

[49] J. L. Wike-Hooley, J. Haveman, and H. S. Reinhold, "The relevance of tumour $\mathrm{pH}$ to the treatment of malignant disease," Radiotherapy \& Oncology, vol. 2, no. 4, pp. 343-366, 1984.

[50] A. De Milito, R. Canese, M. L. Marino et al., "PH-dependent antitumor activity of proton pump inhibitors against human melanoma is mediated by inhibition of tumor acidity," International Journal of Cancer, vol. 127, no. 1, pp. 207-219, 2010.

[51] P. M. J. McSheehy, M. Stubbs, and J. R. Griffiths, "Role of $\mathrm{pH}$ in tumor-trapping of the anticancer drug 5-fluorouracil," Advances in Enzyme Regulation, vol. 40, pp. 63-80, 2000.

[52] R. J. Gillies, N. Raghunand, M. L. Garcia-Martin, and R. A. Gatenby, "pH imaging. A review of $\mathrm{pH}$ measurement methods and applications in cancers," IEEE engineering in medicine and biology magazine : the quarterly magazine of the Engineering in Medicine \& Biology Society, vol. 23, pp. 57-64, 2004.

[53] K. M. Ward, A. H. Aletras, and R. S. Balaban, "A new class of contrast agents for MRI based on proton chemical exchange dependent saturation transfer (CEST)," Journal of Magnetic Resonance, vol. 143, no. 1, pp. 79-87, 2000.

[54] M. L. Garcia-Martin, G. Herigault, C. Remy et al., "Mapping extracellular $\mathrm{pH}$ in rat brain gliomas in vivo by $1 \mathrm{H}$ magnetic resonance spectroscopic imaging: comparison with maps of metabolites," Cancer Research, vol. 61, pp. 6524-6531, 2001.
[55] R. J. Gillies, Z. Liu, and Z. Bhujwalla, "31P-MRS measurements of extracellular $\mathrm{pH}$ of tumors using 3aminopropylphosphonate," American Journal of Physiology-Cell Physiology, vol. 267, pp. C195-C203, 1994.

[56] Z. Zhang, B. Hancock, S. Leen et al., "Compatibility of superparamagnetic iron oxide nanoparticle labeling for $1 \mathrm{H}$ MRI cell tracking with 31P MRS for bioenergetic measurements," NMR in Biomedicine, vol. 23, no. 10, pp. 1166-1172, 2010.

[57] D. Coman, Y. Huang, J. U. Rao et al., "Imaging the intratumoralperitumoral extracellular $\mathrm{pH}$ gradient of gliomas," NMR in Biomedicine, vol. 29, no. 3, pp. 309-319, 2016.

[58] Y. Huang, D. Coman, P. Herman, J. U. Rao, S. Maritim, and F. Hyder, "Towards longitudinal mapping of extracellular $\mathrm{pH}$ in gliomas," NMR in Biomedicine, vol. 29, no. 10, pp. 1364-1372, 2016.

[59] D. Coman, H. K. Trubel, and F. Hyder, "Brain temperature by Biosensor Imaging of Redundant Deviation in Shifts (BIRDS): Comparison between TmDOTP5- and TmDOTMA-," NMR in Biomedicine, vol. 23, no. 3, pp. 277-285, 2010.

[60] D. Coman, H. K. Trubel, R. E. Rycyna, and F. Hyder, "Brain temperature and $\mathrm{pH}$ measured by $1 \mathrm{H}$ chemical shift imaging of a thulium agent," NMR in Biomedicine, vol. 22, no. 2, pp. 229239, 2009.

[61] D. Coman, R. A. De Graaf, D. L. Rothman, and F. Hyder, "In vivo three-dimensional molecular imaging with Biosensor Imaging of Redundant Deviation in Shifts (BIRDS) at high spatiotemporal resolution," NMR in Biomedicine, vol. 26, no. 11, pp. 1589-1595, 2013.

[62] S. Maritim, Y. Huang, D. Coman, and F. Hyder, "Characterization of a lanthanide complex encapsulated with MRI contrast agents into liposomes for biosensor imaging of redundant deviation in shifts (BIRDS)," Journal of Biological Inorganic Chemistry, vol. 19, no. 8, pp. 1385-1398, 2014.

[63] G. Liu, R. Y. Hong, L. Guo, Y. G. Li, and H. Z. Li, "Preparation, characterization and MRI application of carboxymethyl dextran coated magnetic nanoparticles," Applied Surface Science, vol. 257, no. 15, pp. 6711-6717, 2011.

[64] D. Coman, G. E. Kiefer, D. L. Rothman, A. D. Sherry, and F. Hyder, "A lanthanide complex with dual biosensing properties: CEST (chemical exchange saturation transfer) and BIRDS (biosensor imaging of redundant deviation in shifts) with europium DOTA-tetraglycinate," NMR in Biomedicine, vol. 24, no. 10, pp. 1216-1225, 2011.

[65] H. K. F. Trübel, P. K. Maciejewski, J. H. Farber, and F. Hyder, "Brain temperature measured by $1 \mathrm{H}-\mathrm{NMR}$ in conjunction with a lanthanide complex," Journal of Applied Physiology, vol. 94, no. 4, pp. 1641-1649, 2003.

[66] M. W. Brightman, “The Intracerebral Movement of Proteins Injected into Blood and Cerebrospinal Fluid of Mice," Progress in Brain Research, vol. 29, no. C, pp. 19-40, 1968.

[67] A. K. Johnson and P. M. Gross, "Sensory circumventricular organs and brain homeostatic pathways," The FASEB Journal, vol. J7, pp. 678-686, 1993.

[68] O. M. Girard, R. Ramirez, S. McCarty, and R. F. Mattrey, "Toward absolute quantification of iron oxide nanoparticles as well as cell internalized fraction using multiparametric MRI," Contrast Media \& Molecular Imaging, vol. 7, pp. 411-417, 2012.

[69] A. Taylor, A. Herrmann, D. Moss et al., "Assessing the efficacy of nano- and micro-sized magnetic particles as contrast agents for MRI cell tracking," PLoS ONE, vol. 9, no. 6, Article ID e100259, 2014. 
[70] S. H. Crayton and A. Tsourkas, "PH-titratable superparamagnetic iron oxide for improved nanoparticle accumulation in acidic tumor microenvironments," ACS Nano, vol. 5, no. 12, pp. 9592-9601, 2011.

[71] C. Y. Shu, B. G. Sanganahalli, D. Coman, P. Herman, D. L. Rothman, and F. Hyder, "Quantitative $\beta$ mapping for calibrated fMRI," NeuroImage, vol. 126, pp. 219-228, 2016.

[72] F. Kallinowski and P. Vaupel, "Ph distributions in spontaneous and isotransplanted rat tumours," British Journal of Cancer, vol. 58, no. 3, pp. 314-321, 1988.

[73] I. F. Tannock and D. Rotin, "Acid pH in tumors and its potential for therapeutic exploitation," Cancer Research, vol. 49, pp. 43734384, 1989.

[74] F. Hyder and S. M. Hoque, "Brain tumor diagnostics and therapeutics with superparamagnetic ferrite nanoparticles," Contrast Media \& Molecular Imaging, Article ID 6387217, In press.

[75] D. Barbaro, L. Di Bari, V. Gandin et al., "Glucose-coated superparamagnetic iron oxide nanoparticles prepared by metal vapour synthesis are electively internalized in a pancreatic adenocarcinoma cell line expressing GLUT1 transporter," PLoS ONE, vol. 10, no. 4, Article ID e0123159, 2015.

[76] X. H. Shan, P. Wang, F. Xiong et al., "MRI of High-Glucose Metabolism Tumors: a Study in Cells and Mice with 2DG-Modified Superparamagnetic Iron Oxide Nanoparticles," Molecular Imaging and Biology, vol. 18, no. 1, pp. 24-33, 2016.

[77] J. M. Brown and A. J. Giaccia, "The unique physiology of solid tumors: opportunities (and problems) for cancer therapy," Cancer Research, vol. 58, pp. 1408-1416, 1998.

[78] D. J. Chaplin, P. L. Olive, and R. E. Durand, "Intermittent blood flow in a murine tumor: radiobiological effects," Cancer Research, vol. 47, pp. 597-601, 1987.

[79] T. P. Padera, B. R. Stoll, J. B. Tooredman, D. Capen, E. Di Tomaso, and R. K. Jain, "Cancer cells compress intratumour vessels," Nature, vol. 427, article 695, 2004.

[80] H. Uehara, T. Miyagawa, J. Tjuvajev et al., "Imaging experimental brain tumors with 1-aminocyclopentane carboxylic acid and alpha-aminoisobutyric acid: comparison to fluorodeoxyglucose and diethylenetriaminepentaacetic acid in morphologically defined tumor regions," Journal of Cerebral Blood Flow \& Metabolism, vol. 17, pp. 1239-1253, 1997.

[81] S. M. Hoque, Y. Huang, E. Cocco et al., "Improved specific loss power on cancer cells by hyperthermia and MRI contrast of hydrophilic Fex Col-x Fe2 O4 nanoensembles," Contrast Media \& Molecular Imaging, vol. 11, pp. 514-526, 2016.

[82] A. Jordan and K. Maier-Hauff, "Magnetic nanoparticles for intracranial thermotherapy," Journal of Nanoscience and Nanotechnology, vol. 7, pp. 4604-4606, 2007.

[83] B. Thiesen and A. Jordan, "Clinical applications of magnetic nanoparticles for hyperthermia," International Journal of Hyperthermia, vol. 24, no. 6, pp. 467-474, 2008.

[84] F. K. H. van Landeghem, K. Maier-Hauff, A. Jordan et al., "Post-mortem studies in glioblastoma patients treated with thermotherapy using magnetic nanoparticles," Biomaterials, vol. 30, no. 1, pp. 52-57, 2009.

[85] K. Maier-Hauff, R. Rothe, and R. Scholz, "Intracranial thermotherapy using magnetic nanoparticles combined with external beam radiotherapy: results of a feasibility study on patients with glioblastoma multiforme," Journal of Neuro-Oncology, vol. 81, no. 1, pp. 53-60, 2007.

[86] J. W. M. Bulte and D. L. Kraitchman, "Monitoring cell therapy using iron oxide MR contrast agents," Current Pharmaceutical Biotechnology, vol. 5, no. 6, pp. 567-584, 2004.
[87] H. E. Daldrup-Link, R. Meier, M. Rudelius et al., "In vivo tracking of genetically engineered, anti-HER2/neu directed natural killer cells to HER2/neu positive mammary tumors with magnetic resonance imaging," European Radiology, vol. 15, no. 1, pp. 4-13, 2005.

[88] H. E. Daldrup-Link, M. Rudelius, G. Piontek et al., "Migration of iron oxide-labeled human hematopoietic progenitor cells in a mouse model: In vivo monitoring with 1.5-T ME imaging equipment," Radiology, vol. 234, no. 1, pp. 197-205, 2005.

[89] L. Kostura, D. L. Kraitchman, A. M. Mackay, M. F. Pittenger, and J. M. W. Bulte, "Feridex labeling of mesenchymal stem cells inhibits chondrogenesis but not adipogenesis or osteogenesis," NMR in Biomedicine, vol. 17, no. 7, pp. 513-517, 2004.

[90] C. H. Fan, C. Y. Ting, H. J. Lin et al., "SPIO-conjugated, doxorubicin-loaded microbubbles for concurrent MRI and focused-ultrasound enhanced brain-tumor drug delivery," Biomaterials, vol. 34, no. 14, pp. 3706-3715, 2013.

[91] P. Zhang, L. Hu, Q. Yin, L. Feng, and Y. Li, "Transferrinmodified c[RGDfK]-paclitaxel loaded hybrid micelle for sequential blood-brain barrier penetration and glioma targeting therapy," Molecular Pharmaceutics, vol. 9, no. 6, pp. 1590-1598, 2012.

[92] P. Zhang, L. Hu, Q. Yin, Z. Zhang, L. Feng, and Y. Li, "Transferrin-conjugated polyphosphoester hybrid micelle loading paclitaxel for brain-targeting delivery: Synthesis, preparation and in vivo evaluation," Journal of Controlled Release, vol. 159, no. 3, pp. 429-434, 2012.

[93] Z. Zhen, W. Tang, H. Chen et al., "RGD-modified apoferritin nanoparticles for efficient drug delivery to tumors," ACS Nano, vol. 7, no. 6, pp. 4830-4837, 2013.

[94] Z. Zhen, W. Tang, C. Guo et al., "Ferritin nanocages to encapsulate and deliver photosensitizers for efficient photodynamic therapy against cancer," ACS Nano, vol. 7, no. 8, pp. 6988-6996, 2013.

[95] L. Qin, C.-Z. Wang, H.-J. Fan et al., "A dual-targeting liposome conjugated with transferrin and arginine-glycine-aspartic acid peptide for glioma-targeting therapy," Oncology Letters, vol. 8, no. 5, pp. 2000-2006, 2014.

[96] J. U. Rao, U. F. H. Engelke, F. C. G. J. Sweep et al., "Genotype-specific differences in the tumor metabolite profile of pheochromocytoma and paraganglioma using untargeted and targeted metabolomics," The Journal of Clinical Endocrinology \& Metabolism, vol. 100, no. 2, pp. E214-E222, 2015.

[97] T. Inai, M. Mancuso, H. Hashizume et al., "Inhibition of vascular endothelial growth factor (VEGF) signaling in cancer causes loss of endothelial fenestrations, regression of tumor vessels, and appearance of basement membrane ghosts," The American journal of pathology, vol. 165, pp. 35-52, 2004.

[98] R. J. Gillies and R. A. Gatenby, "Adaptive landscapes and emergent phenotypes: Why do cancers have high glycolysis?" Journal of Bioenergetics and Biomembranes, vol. 39, no. 3, pp. 251-257, 2007.

[99] R. J. Gillies, I. Robey, and R. A. Gatenby, "Causes and consequences of increased glucose metabolism of cancers," Journal of Nuclear Medicine, vol. 49, supplement 2, pp. 24S-42S, 2008.

[100] I. F. Robey, B. K. Baggett, N. D. Kirkpatrick et al., "Bicarbonate increases tumor $\mathrm{pH}$ and inhibits spontaneous metastases," Cancer Research, vol. 69, no. 6, pp. 2260-2268, 2009.

[101] A. Ibrahim-Hashim, D. Abrahams, P. M. Enriquez-Navas, K. Luddy, R. A. Gatenby, and R. J. Gillies, "Tris-base buffer: a promising new inhibitor for cancer progression and metastasis," Cancer Medicine, vol. 6, pp. 1720-1729, 2017. 
[102] J. U. Rao, D. Coman, J. J. Walsh, M. M. Ali, Y. Huang, and F. Hyder, "Temozolomide arrests glioma growth and normalizes intratumoral extracellular pH," Scientific Reports, vol. 7, article 7865, 2017.

[103] K. -. C. Chu, Y. Xu, J. A. Balschi, and C. S. Springer, "Bulk magnetic susceptibility shifts in nmr studies of compartmentalized samples: use of paramagnetic reagents," Magnetic Resonance in Medicine, vol. 13, no. 2, pp. 239-262, 1990.

[104] A. D. Sherry, P. Caravan, and R. E. Lenkinski, "Primer on gadolinium chemistry," Journal of Magnetic Resonance Imaging, vol. 30, no. 6, pp. 1240-1248, 2009.

[105] E. Ledneva, S. Karie, V. Launay-Vacher, N. Janus, and G. Deray, "Renal safety of gadolinium- Based contrast media in patients with chronic renal insufficiency," Radiology, vol. 250, no. 3, pp. 618-628, 2009.

[106] J. Zhou, T. R. Patel, R. W. Sirianni et al., "Highly penetrative, drug-loaded nanocarriers improve treatment of glioblastoma," Proceedings of the National Acadamy of Sciences of the United States of America, vol. 110, no. 29, pp. 11751-11756, 2013. 


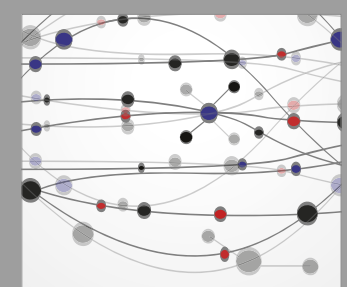

The Scientific World Journal
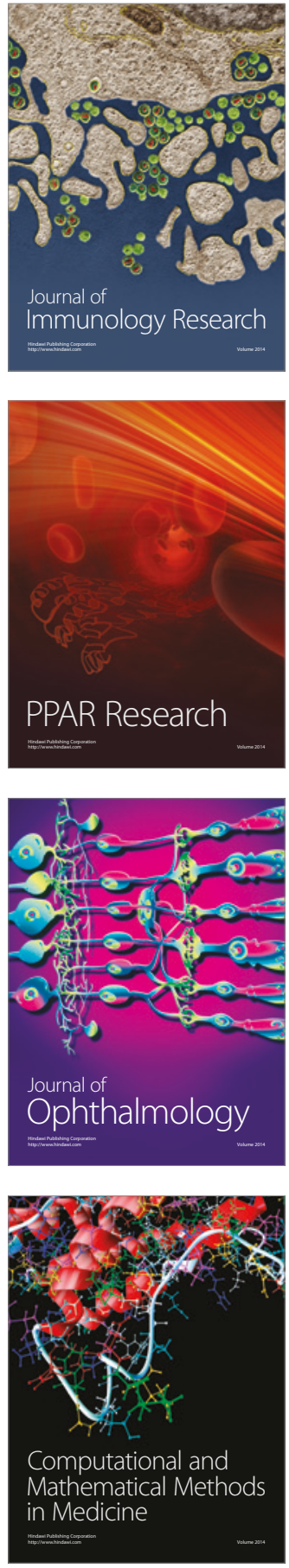

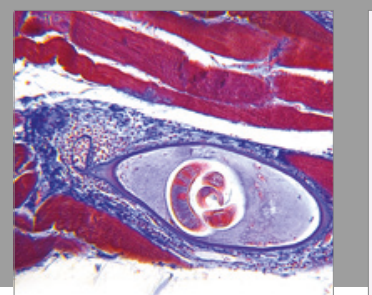

Gastroenterology Research and Practice
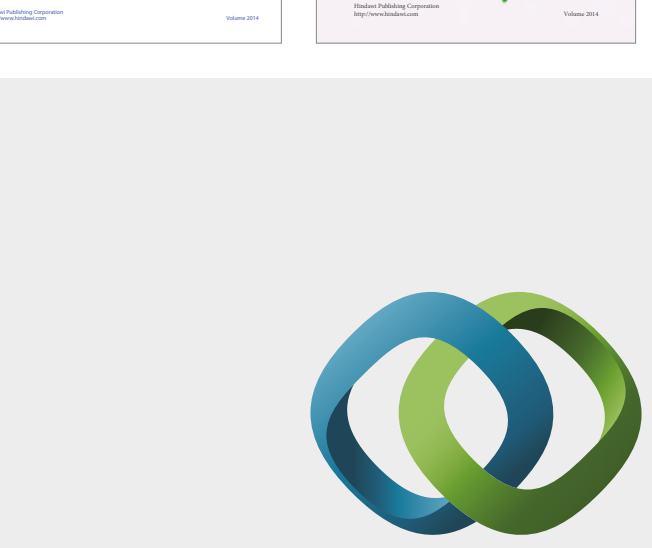

\section{Hindawi}

Submit your manuscripts at

https://www.hindawi.com
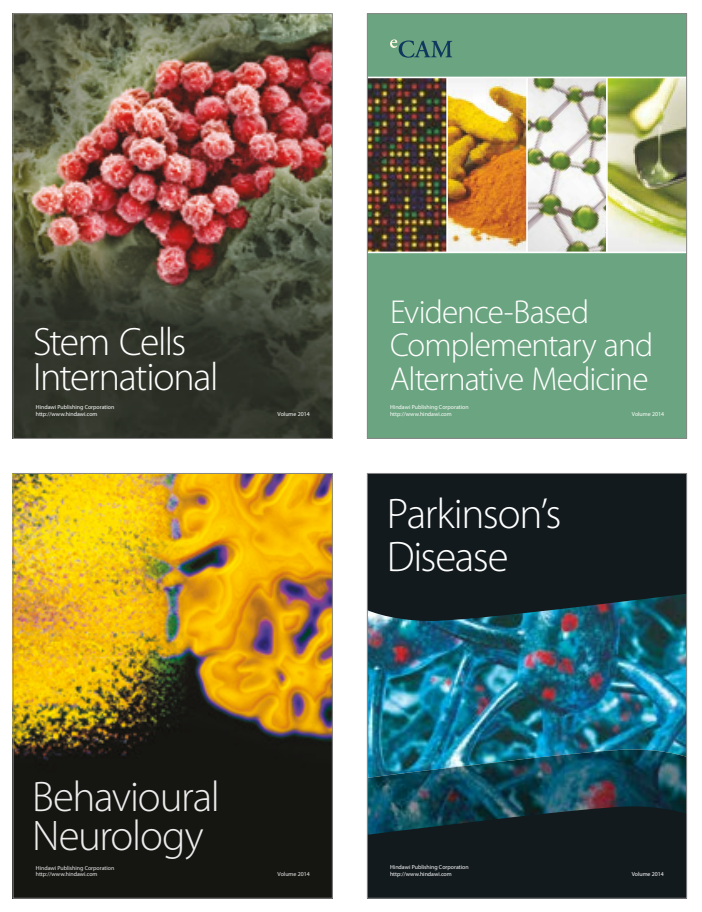
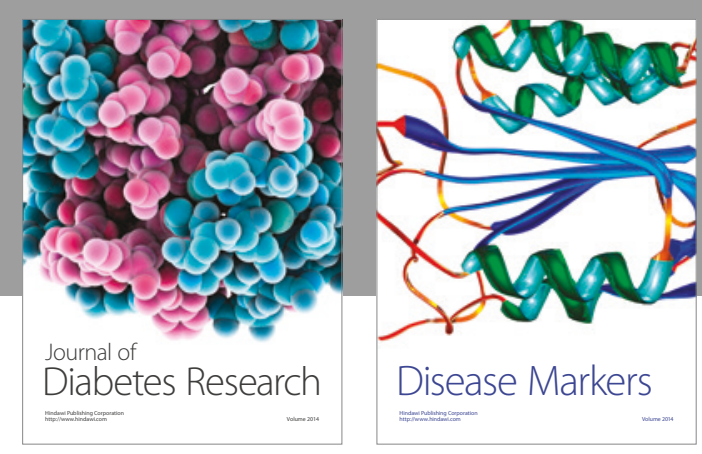

Disease Markers
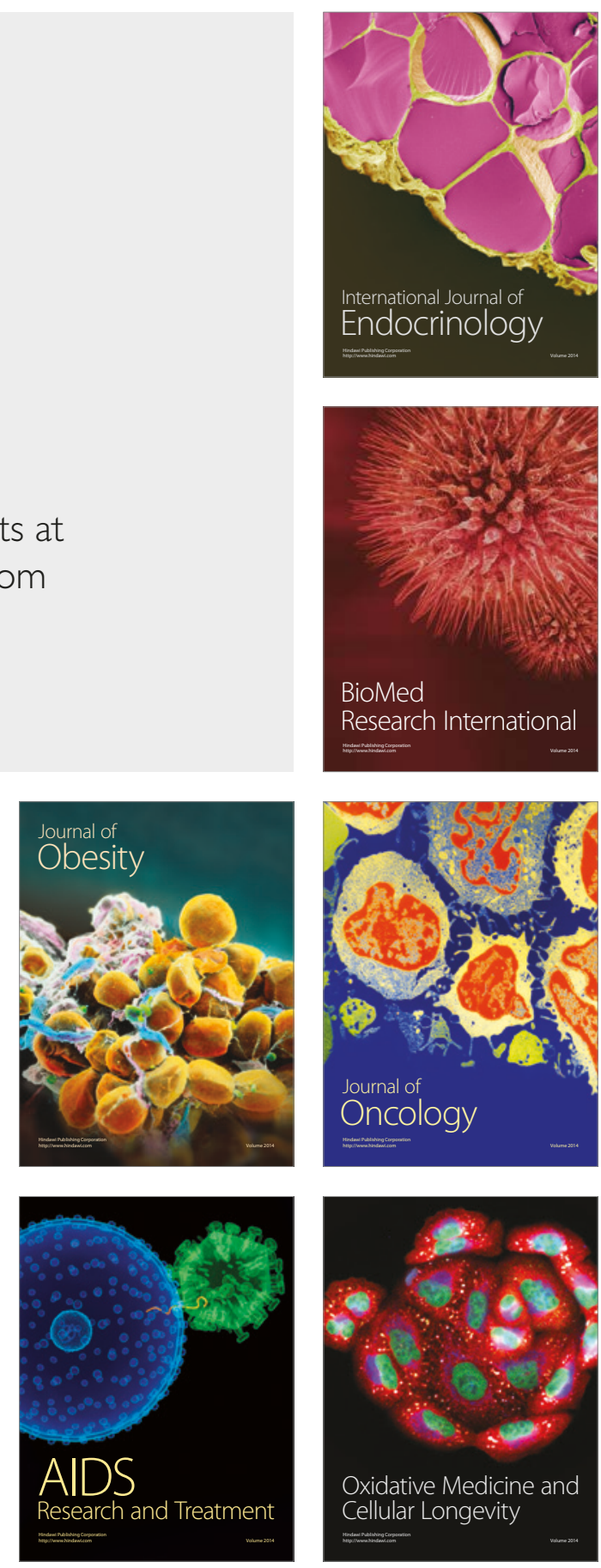